\title{
Reversible High Temperature Cells for Power Generation and Hydrogen Production using Mixed Ionic Electronic Conducting Solid Electrolytes
}

\author{
Anil V. Virkar ${ }^{1, *}$ and Greg Tao ${ }^{2}$ \\ ${ }^{1}$ Department of Materials Science and Engineering \\ University of Utah \\ Salt Lake City, Utah 84112 \\ USA \\ and \\ ${ }^{2}$ Materials and Systems Research, Inc. \\ 5395 West 700 South \\ Salt Lake City, Utah 84104 \\ USA \\ *Contributing Author \\ Email: anil.virkar@utah.edu
}




\begin{abstract}
High temperature solid oxide cells can be used both as fuel cells (SOFC) for power generation and as electrolyzer cells (SOEC) for hydrogen generation. Such cells are called reversible cells. They are typically made using solid electrolytes which exhibit purely ionic conduction and negligible electronic conduction. In this work reversible cells made of mixed ionic electronic conducting (MIEC) materials with significant electronic conduction as electrolyte are investigated. It is shown that MIEC cells can be designed to operate as efficiently as those made of a purely ionic conducting electrolyte (e.g. YSZ). Thus, in SOFC mode, suitably designed MIEC electrolyte cells consume fuel at the same rate, deliver the same power and release the same amount of joule heat as purely ion conducting electrolyte cells. Similarly, in SOEC mode, MIEC electrolyte cells consume water vapor at the same rate, generate hydrogen at the same rate, consume the same electrical power and release the same joule heat as purely ionic conducting electrolyte cells. Experimental results are presented on SOEC stacks made of YSZ and MIEC electrolyte cells. The MIEC electrolyte cells exhibit more stable operation compared to purely ion conducting electrolyte (YSZ) cells. The improved durability of MIEC electrolyte cells is attributed to the smoothening of the chemical potential of oxygen, $\mu_{O_{2}}(x)$, variation in the electrolyte. The analysis also shows that highly active electrodes should lower the tendency for degradation even with a purely ion conducting electrolyte cell.
\end{abstract}

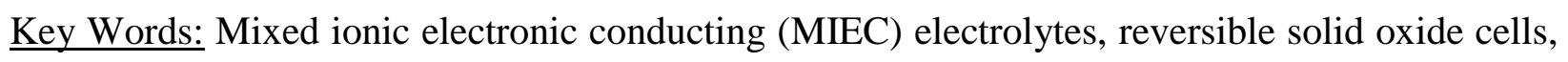
electrolysis, fuel cells. 


\section{Introduction}

Solid oxide fuel cells (SOFC) and solid oxide electrolyzer cells (SOEC) are typically made using solid electrolytes exhibiting purely ionic (often for oxygen ions) conductivity and negligible electronic conductivity. Much of the work over the past several decades, going back to the 1960's, has sought materials for solid electrolytes with the highest possible ionic transference number. The rationale is that if the electrolyte is a purely ionic conductor, negligible electronic leakage occurs through the electrolyte leading to higher efficiency. In the case of SOFC, any internal electronic leakage leads to part of the fuel being consumed without generating electrical energy - and thus leading to loss of efficiency. In the case of SOEC, part of the input electrical energy is degraded as heat through the electronic component of the electrolyte. When an SOFC or an SOEC is actually operating, however, some of the potential useful work is naturally degraded as heat (joule heating) regardless of whether the electrolyte is a purely ionic conductor or exhibits significant electronic conductivity. That is, in accord with thermodynamics, it is understood that some of the potential work must be degraded as heat to generate work at practically useful rates (generate power at some useful rate). In the case of SOEC also, it is understood that some of the supplied electrical energy must be converted (degraded) into heat to generate hydrogen at some practical rates. The main performance-related objective is to design a device such that it operates as efficiently as possible while delivering power at a useful rate or 
generating hydrogen at a useful rate. When a cell is operating at some finite current, $I_{L}$, the rate of joule heating is given by $I_{L}^{2} R_{\text {cell }}$ which is a loss of potential work into heat, where $R_{\text {cell }}$ is the cell resistance - assumed to be a purely ionic conductor.

Now suppose the cell electrolyte is not a purely ionic conductor but is a mixed ionic electronic conductor (MIEC) such that its ionic and electronic resistances are respectively $R_{i}$ and $R_{e}$ where $R_{e} \neq \infty$, but $R_{e}>R_{i}$. When such a cell is operating, the joule heating is given by $I_{i}^{2} R_{i}+I_{e}^{2} R_{e}$ where $I_{i}$ is the ionic current flowing through the cell and $I_{e}$ is the electronic current flowing through the cell. It is easy to show that the measured current is given by $I_{L}=\mp\left|I_{i}\right|+I_{e}$. An important question concerns if it is possible for a purely ion conducting cell and an MIEC cell to exhibit the same performance characteristics at some (which in general will be different for the two cells) operating conditions. If this is possible, the other important questions are: (1) What should be the respective cell parameters under which an MIEC cell exhibits the same performance characteristics as a purely ion conducting electrolyte cell? (2) Is there any benefit to using an MIEC electrolyte cell instead of a purely ion conducting cell? In our work on the stability of electrochemical devices, we have previously demonstrated, both theoretically and experimentally, that MIEC cells exhibit greater durability than cells made with purely ion conducting electrolytes [1-3]. Specifically, it has been shown experimentally that MIEC cells are less prone to issues such as electrode delamination [3]. Thus, the second question to an extent has been addressed in our prior work [1-3]. The primary objective of this work is to examine the first question; namely whether it is possible to design cells with MIEC electrolytes which can operate as efficiently as cells made with purely ion conducting electrolytes. 


\section{Theory}

In most solid oxide cells, the anode and the cathode are graded with regard to composition, porosity, and microstructure. Thus, a realistic representation of a cell in any modeling study requires an analysis of cells having multiple layers with differing electrical and gas transport properties. This must also then reflect in the corresponding equivalent circuit which may be used to analyze the electrical response of such a cell when using techniques such as electrochemical impedance spectroscopy (EIS). However, often such detailed information about the number of layers, microstructures, porosities, pore sizes, compositions and the properties of various layers is not reported in the literature. Thus, it is often not possible to satisfactorily model experimentally measured voltage vs. current curves in which all processes occurring in multiple layers are accounted for accurately, since the properties (e.g. porosity, pore size, conductivity, etc.) can be different in different layers. For this reason, practical analysis is often based on some equivalent circuit. The simplest equivalent circuit at a minimum has three segments: (a) Cathode, (b) Electrolyte, and (c) Anode. Also, if the concentration polarization can be neglected and the activation polarization can be described by the linearized Butler-Volmer equation, the equivalent circuit may be given in the most simplified form, yet inclusive of the key features which allow a realistic representation under at least low current densities. In what follows, such a simplified 
approach is used. This allows one to obtain relevant equations in a simple, yet in a fully analytical form.

$\underline{\text { An Equivalent Circuit for a Solid Oxide Cell including Electrodes: Figure } 1 \text { shows a simplified }}$ equivalent circuit for a solid oxide cell in steady state in which the polarization resistances at both electrodes are included. The polarization resistance at the oxygen electrode is given by $R_{i(c)}$ and the polarization resistance at the fuel electrode is given by $R_{i(a)}$. These two in principle can be given in terms of the corresponding exchange current densities. The ionic resistance of the electrolyte is given by $R_{i(e l)}$. Thus, the net area specific ionic resistance of the cell is given by

$$
R_{i}=R_{i(c)}+R_{i(e l)}+R_{i(a)}
$$

In EIS this reflects as an intermediate frequency intercept. As shown previously [1], the electronic resistance cannot be set to zero mathematically for either the electrolyte or the electrolyte/electrode interfaces, since this amounts to a violation of the local thermodynamic equilibrium (chemical potentials are undetermined) [1,4]. The electronic resistances are given by $R_{e(c)}, R_{e(a)}$ and $R_{e(e l)}$, where $R_{e(c)}$ is the electronic resistance of the oxygen electrode/electrolyte interface (resistance associated with the transport of electrons across the oxygen electrode/electrolyte interface), $R_{e(a)}$ is the electronic resistance of the fuel electrode/electrolyte interface (resistance associated with the transport of electrons across the fuel electrode/electrolyte interface) and $R_{e(e l)}$ is the electronic resistance of the electrolyte. 
These electronic resistances can be large compared to the ionic resistances, but are not mathematically infinite. The net electronic resistance of the cell is

$$
R_{e}=R_{e(c)}+R_{e(e l)}+R_{e(a)}
$$

For a purely ion conducting electrolyte, $\quad R_{i(e l)}<<R_{e(e l)}$ and also

$$
R_{i}=R_{i(c)}+R_{i(e l)}+R_{i(a)}<<R_{e}=R_{e(c)}+R_{e(e l)}+R_{e(a)}
$$

The $R_{e}$ is thus not reflected in the typical EIS spectra. It can be determined for the electrolyte by a separate technique such as the blocking electrode method. It can also be estimated using probes embedded within the electrolyte, although this requires careful fabrication of cells with embedded probes [5]. As shown previously [1,2], the electronic resistance must appear as a parallel resistor in each segment of the equivalent circuit as shown in Figure 1. That is, if the equivalent circuit is discretized into $N$ segments, the ionic and the electronic resistors must appear in each segment in parallel and joined at the nodes [1]. In some reported equivalent circuits, the electronic resistance of the cell has been included as a parallel rail across the entire cell equivalent circuit. This, however, violates the criterion of local thermodynamic equilibrium $[1,4]$. This is discussed below.

Local chemical equilibrium for oxygen means the reaction 


$$
O^{2-} \rightarrow \frac{1}{2} O_{2}+2 e^{\prime}
$$

must be in equilibrium inside the electrolyte at any position $x$, which means [6]

$$
\tilde{\mu}_{O^{2-}}(x)=\frac{1}{2} \mu_{O_{2}}(x)+2 \tilde{\mu}_{e}(x)
$$

where $\tilde{\mu}_{O^{2-}}(x)$ is the electrochemical potential of $\mathrm{O}^{2-}$ ions at $x, \tilde{\mu}_{e}(x)$ is the electrochemical potential of electrons at $x$ and $\mu_{O_{2}}(x)$ is the chemical potential of oxygen at $x$. In addition, each ionic segment must have an internal Nernst potential which represents the difference in the oxygen chemical potentials, $\mu_{O_{2}}$, across that segment $[1,2]$. This further means the chemical potential of oxygen is a single-valued function of position, namely, $\mu_{O_{2}}(x)$ (the chemical potentials of all other species are also single-valued functions of position). These requirements have their origin in the laws of thermodynamics and the existence of local equilibrium, which is the fundamental assumption in all global equilibrium thermodynamics, in all linear nonequilibrium (and thus non-global) thermodynamics and in much of the nonlinear non-equilibrium thermodynamics [7-9].

In the present case, we have discretized the cell equivalent circuit into three segments. In many cases of interest, equation (3) holds, which would be the case with say yttria-stabilized zirconia (YSZ) as the electrolyte, which is a purely oxygen ion conductor with negligible electronic conductivity (transference number $t_{i}>0.99$ ). In such a case the cell resistance can be considered to be purely ionic for the purposes of calculating the current-voltage characteristics. However, as 
emphasized earlier and described elsewhere, if the objective is to determine the chemical potentials inside the membrane (which determines durability) then the electronic resistance cannot be regarded as being infinite (even though it may be very large) [1,2]. The present focus is primarily on the performance of cells. Thus, for the most part simplified equivalent circuits are used in what follows.

The equivalent circuit shown in Figure 1 is the simplest one that is necessary and sufficient when the objective is to estimate the spatial distribution of oxygen chemical potential, $\mu_{O_{2}}$, through the cell. The experimentally known values are the oxygen chemical potentials at the electrodes; $\mu_{\mathrm{O}_{2}}^{I}$ at the oxygen electrode (cathode for SOFC) and $\mu_{\mathrm{O}_{2}}^{I I}$ at the fuel electrode (anode for SOFC). Then, the cell Nernst potential is given by (assumes that the concentration polarization is small)

$$
E_{N}=\frac{\left(\mu_{O_{2}}^{I}-\mu_{O_{2}}^{I I}\right)}{4 F}
$$

However, the chemical potential of oxygen varies through the electrolyte. If the oxygen chemical potential in the electrolyte very close to the oxygen electrode is given by $\mu_{O_{2}}^{c}$, then the Nernst potential corresponding to the oxygen electrode/electrolyte interface is given by

$$
E_{N(c)}=\frac{\left(\mu_{O_{2}}^{I}-\mu_{O_{2}}^{c}\right)}{4 F}
$$


Similarly, if the oxygen chemical potential in the electrolyte very close to the fuel electrode is given by $\mu_{O_{2}}^{a}$, then the Nernst potential corresponding to the fuel electrode/electrolyte interface is given by

$$
E_{N(a)}=\frac{\left(\mu_{O_{2}}^{a}-\mu_{O_{2}}^{I I}\right)}{4 F}
$$

Thus, the Nernst potential across the electrolyte (just inside the electrolyte/electrode interfaces) is given by

$$
E_{N(e l)}=\frac{\left(\mu_{O_{2}}^{c}-\mu_{O_{2}}^{a}\right)}{4 F}
$$

If the electronic and the ionic conductivities of the electrolyte are constant (not positiondependent), then the oxygen chemical potential varies linearly through the electrolyte between $\mu_{O_{2}}^{c}$ and $\mu_{O_{2}}^{a}$.

The total Nernst potential across the cell is given by

$$
E_{N}=\frac{\left(\mu_{O_{2}}^{I}-\mu_{O_{2}}^{I I}\right)}{4 F}=\frac{\left(\mu_{O_{2}}^{I}-\mu_{O_{2}}^{c}\right)}{4 F}+\frac{\left(\mu_{O_{2}}^{c}-\mu_{O_{2}}^{a}\right)}{4 F}+\frac{\left(\mu_{O_{2}}^{a}-\mu_{O_{2}}^{I I}\right)}{4 F}=E_{N(c)}+E_{N(e l)}+E_{N(a)}
$$

In steady state, both the ionic and the electronic currents are independent of time and position (one dimensional problem). Thus, for estimating the overall performance characteristics of the cell, it is not necessary to use the segmented equivalent circuit given in Figure 1 (which is 
needed, however, for estimating the oxygen chemical potential variation) $[1,2]$. In steady state the ionic current is uniform through all three ionic segments and is given by

$$
E_{A}=E_{N(c)}+E_{N(e l)}+E_{N(a)}-I_{i}\left(R_{i(c)}+R_{i(e l)}+R_{i(a)}\right)=E_{N}-I_{i} R_{i}
$$

The electronic current is also uniform through all three electronic segments and is given by

$$
E_{A}+I_{e}\left(R_{e(c)}+R_{e(e l)}+R_{e(a)}\right)=E_{A}+I_{e} R_{e}=0
$$

Thus, in steady state, $R_{i(c)}, R_{i(a)}$ and $R_{i(e l)}$ can be lumped into a single $R_{i}$, given by equation (1), and $R_{e(c)}, R_{e(a)}$ and $R_{e(e l)}$ can be lumped into a single $R_{e}$, given by equation (2). In such a case a further simplified equivalent circuit shown in Figure 2 can be used. Thus, in this equivalent circuit, the net ionic resistance of the cell (which includes the two electrode activation polarization resistances) is given by equation (1). The net electronic resistance of the cell (which includes electrode/electrolyte interfaces) is given by equation (2), and the net Nernst potential is again given by equations (6) and (10).

We now consider two types of cells. In one cell, the electrolyte is a purely ionic conductor and the electronic resistance can be ignored in the calculation of performance characteristics of the cell. Such could be the cells made with YSZ as the electrolyte, for example. In the other cell, the electronic resistance of the cell is not too large compared to the ionic resistance. That is, $R_{e}$ is not too large compared to $R_{i}$. In such a case, the electronic resistance cannot be ignored even for the calculation of the performance characteristics. Such could be the case with rare earth oxide 
doped ceria as the electrolyte, or a YSZ electrolyte doped with $\mathrm{CeO}_{2}$. In what follows, we discuss these two types of cells operated in SOEC and SOFC modes.

$\underline{\text { Solid Oxide Cells with a Purely Ion Conducting Solid Electrolyte (e.g. YSZ) and a Mixed Ionic }}$ and Electronic Conducting (MIEC) Solid Electrolyte:

Figure 3(a) shows a simplified equivalent circuit in steady state for a cell with a purely ion conducting electrolyte. The parallel electronic resistor, $R_{e(1)}$, (shown by broken lines) is assumed to be very large (and thus can be neglected in the calculation of the current-voltage characteristics). Figure 3(b) shows a simplified equivalent circuit in steady state for a cell with an MIEC electrolyte in which the parallel electronic resistor, $R_{e(2)}$, is not infinitely large compared to the ionic resistance, $R_{i(2)}$, and thus must be included in the calculation of the current-voltage characteristics.

The ionic current flowing through the cell of a purely ion conducting electrolyte is

$$
I_{i(1)}=\frac{E_{N}-E_{A(1)}}{R_{i(1)}}=I_{L(1)}
$$

which is also the measured current in the external circuit, $I_{L(1)}$. In SOEC mode $E_{A(1)}>E_{N}$ and $I_{L(1)}<0$. In SOFC mode, $E_{A(1)}<E_{N}$ and $I_{L(1)}>0$.

The ionic current flowing through the cell of an MIEC electrolyte is given by 


$$
I_{i(2)}=\frac{E_{N}-E_{A(2)}}{R_{i(2)}}
$$

In SOEC mode, $I_{i(2)}<0$, and in SOFC mode, $I_{i(2)}>0$.

The electronic current flowing through the MIEC cell is given by

$$
I_{e(2)}=-\frac{E_{A(2)}}{R_{e(2)}}
$$

Thus, the net current measured in the external circuit for the MIEC cell is

$$
I_{L(2)}=I_{i(2)}+I_{e(2)}=\frac{E_{N}-E_{A(2)}}{R_{i(2)}}-\frac{E_{A(2)}}{R_{e(2)}}
$$

The ionic and the electronic currents are parallel to each other in the SOEC mode, but are antiparallel in the SOFC mode [1].

The condition that both cells exhibit the same hydrogen generation rate (SOEC) or consumption rate (SOFC) is given by

$$
I_{i(1)}=\frac{E_{N}-E_{A(1)}}{R_{i(1)}}=I_{i(2)}=\frac{E_{N}-E_{A(2)}}{R_{i(2)}}
$$

That is, the ionic currents in the two cells are identical. 
The condition that both cells exhibit identical joule heating is given by

$$
\frac{\left(E_{N}-E_{A(1)}\right)^{2}}{R_{i(1)}}=\frac{\left(E_{N}-E_{A(2)}\right)^{2}}{R_{i(2)}}+\frac{E_{A(2)}^{2}}{R_{e(2)}}
$$

Finally, the condition that both cells 'generate' identical electrical power (SOFC) or 'consume' identical electrical power (SOEC), is given by

$$
E_{A(1)} I_{L(1)}=E_{A(2)} I_{L(2)}
$$

If these three conditions (one is redundant since there are three equations but only two unknowns, $E_{A}(1)$ and $\left.E_{A}(2)\right)$ are satisfied, it means the two cells exhibit identical performance characteristics under the corresponding sets of operating conditions.

From the preceding equations, it can be shown that $E_{A(2)}$ is given by the solution to the quadratic equation

$$
a E_{A(2)}^{2}+b E_{A(2)}+c=0
$$

where the coefficients are given by 


$$
\begin{aligned}
& a=\left(R_{i(2)}-R_{i(1)}\right) R_{e(2)}+R_{i(2)}^{2} \\
& b=2 E_{N} R_{e(2)}\left(R_{i(1)}-R_{i(2)}\right)
\end{aligned}
$$

and

$$
c=E_{N}^{2} R_{e(2)}\left(R_{i(2)}-R_{i(1)}\right)
$$

Thus, $E_{A(2)}$ is given by

$$
E_{A(2)}=\frac{-b \pm \sqrt{b^{2}-4 a c}}{2 a}
$$

The relationship between $E_{A(1)}$ and $E_{A(2)}$ is given by

$$
E_{A(1)}=E_{N}+\left(E_{A(2)}-E_{N}\right) \frac{R_{i(1)}}{R_{i(2)}}
$$

It is understood that there may be certain values of parameters for which there may be no situation possible under which the preceding conditions are satisfied. This will reflect in the calculations as unrealistic values, for instance, calculated non real (complex) numbers for the applied voltages. Note: We are only considering the DC processes (steady state) so there are no reactive components of current or voltage. 
It is important to note that in the preceding, the ionic resistances include the electrode polarization resistances. Thus, there can be a large difference in $R_{i(1)}$ and $R_{i(2)}$ even though the electrolyte materials may not be vastly different. For example, suppose the purely ionic conducting membrane is made of YSZ. The corresponding ionic resistance includes cathode and anode polarization resistances. That is

$$
R_{i(1)}=R_{i(1)}^{c}+R_{i(1)}^{e l}+R_{i(1)}^{a}
$$

Now suppose the mixed ionic electronic conducting electrolyte is made of ceria-doped YSZ. Now

$$
R_{i(2)}=R_{i(2)}^{c}+R_{i(2)}^{e l}+R_{i(2)}^{a}
$$

Thus, we expect $R_{i(1)}^{e l} \cong R_{i(2)}^{e l} \quad$ (similar ionic conductivities for YSZ and ceria-doped YSZ). However, we may still have $R_{i(1)}>R_{i(2)}$ if the polarization resistances are quite different. Based on the experimental results discussed later, this appears to be the case in the present work. The presence of dissolved ceria is believed to be lower the charge transfer resistance. The beneficial effect of a ceria layer in lowering the polarization resistance has been reported in the literature. Thus, in our calculations, we will assume $R_{i(1)}>R_{i(2)}$. Other possibilities for the electrolyte include Sr- and Mg-doped $\mathrm{LaGaO}_{3}$ (LSGM), which can be additionally doped with a transition metal to increase its electronic conductivity. In such a case, $R_{i(2)}^{e l}<R_{i(1)}^{e l}$. 
An Illustrative Example: $\mathrm{An}_{2}$ O-Rich Fuel Gas: In what follows, we will choose some values of the various parameters for the two types of cells for illustrative purposes. The values selected are arbitrary, yet in the range of values (at a cell level) reported in many studies. We assume the fuel gas composition as $70 \% \mathrm{H}_{2} \mathrm{O}+30 \% \mathrm{H}_{2}$. We assume the cells are operated at $800^{\circ} \mathrm{C}$ and the partial pressure of oxygen in the oxidant is such that the Nernst voltage, $E_{N}$, is $0.9 \mathrm{~V}$ (which corresponds to the oxidant as air). The various parameters selected are given below:

$R_{i(1)}=0.5 \Omega \mathrm{cm}^{2} \quad R_{i(2)}=0.2 \Omega \mathrm{cm}^{2} \quad R_{e(2)}=1.5 \Omega \mathrm{cm}^{2}$. This means the ionic transference of the MIEC cell is $t_{i(2)}=\frac{R_{e(2)}}{R_{i(2)}+R_{e(2)}}=0.8824$.

Using these values, we have

$$
\begin{aligned}
& a=\left(R_{i(2)}-R_{i(1)}\right) R_{e(2)}+R_{i(2)}^{2}=-0.41 \Omega^{2} \mathrm{~cm}^{4} \\
& b=2 E_{N} R_{e(2)}\left(R_{i(1)}-R_{i(2)}\right)=0.81 \mathrm{~V} \Omega^{2} \mathrm{~cm}^{4}
\end{aligned}
$$

and

$$
c=E_{N}^{2} R_{e(2)}\left(R_{i(2)}-R_{i(1)}\right)=-0.3645 \mathrm{~V}^{2} \Omega^{2} \mathrm{~cm}^{4}
$$

Solution to equation (20) gives

$$
E_{A(2)}=\frac{-b \pm \sqrt{b^{2}-4 a c}}{2 a}
$$


The two values of $E_{A(2)}$ are

$$
E_{A(2)}=1.2823 \mathrm{~V} \text { and } E_{A(2)}=0.6933 \mathrm{~V}
$$

In SOEC mode, the applied voltage must be greater than the Nernst voltage for a finite, nonzero hydrogen generation rate. Thus, the applied voltage is $E_{A(2)}=1.2823 \mathrm{~V}$. The corresponding applied voltage for the cell made of purely ionic conducting electrolyte is

$$
E_{A(1)}=E_{N}+\left(E_{A(2)}-E_{N}\right) \frac{R_{i(1)}}{R_{i(2)}}=1.8558 \mathrm{~V}
$$

Note that the ionic current densities are

$$
I_{i(1)}=\frac{E_{N}-E_{A(1)}}{R_{i(1)}}=-1.9115 \mathrm{Acm}^{-2}=I_{i(2)}=\frac{E_{N}-E_{A(2)}}{R_{i(2)}}=-1.9115 \mathrm{Acm}^{-2}
$$

which correspond to the hydrogen generation rates. Note also that

$$
I_{e(2)}=-\frac{E_{A(2)}}{R_{e(2)}}=-0.8549 \mathrm{Acm}^{-2}
$$

(the ionic and the electronic currents are parallel in the SOEC mode) [1].

The joule heating rates in the two cells are given by 


$$
\frac{\left(E_{N}-E_{A(1)}\right)^{2}}{R_{i(1)}}=1.8271 \mathrm{Wcm}^{-2}=\frac{\left(E_{N}-E_{A(2)}\right)^{2}}{R_{i(2)}}+\frac{E_{A(2)}^{2}}{R_{e(2)}}=1.827 \mathrm{Wcm}^{-2}
$$

Finally, the electrical energy generation (which is consumption in the SOEC mode) rates for the two cells must also be the same. Thus, we must have

$$
E_{A(1)} I_{L(1)}=E_{A(2)} I_{L(2)}
$$

For cell 1 the measured current (load current) is also the ionic current. For cell 2 the measured load current is given by

$$
I_{L(2)}=I_{i(2)}+I_{e(2)}=-2.7664 \mathrm{Acm}^{-2}
$$

Thus, note that

$$
E_{A(1)} I_{L(1)}=1.8558 \times(-1.9115)=-3.5474 \mathrm{Wcm}^{-2}=E_{A(2)} I_{L(2)}=1.2823 \times(-2.7664)=-3.5474 \mathrm{Wcm}^{-2}
$$

The preceding shows that both cells, one made of a purely ion conducting electrolyte (e.g. YSZ) and the other made of an MIEC electrolyte with significant electronic conductivity (such as YSZ doped with ceria), cell ionic transference number of $\sim 0.88$, can be operated such that they consume the same amount of electricity, generate the same amount of joule heat, and generate 
the same amount of hydrogen. Thus, insofar as the performance characteristics are concerned both cells exhibit identical behavior (when operated at the respective sets of conditions).

Now suppose we choose the second solution, namely $E_{A(2)}=0.6933 \mathrm{~V}$. This is the SOFC mode since $E_{A(2)}<E_{N}$.

Now, the corresponding $E_{A(1)}$ is given by

$$
E_{A(1)}=E_{N}+\left(E_{A(2)}-E_{N}\right) \frac{R_{i(1)}}{R_{i(2)}}=0.3833 \mathrm{~V}
$$

The corresponding ionic currents are given by

$$
I_{i(1)}=\frac{E_{N}-E_{A(1)}}{R_{i(1)}}=1.0334 \mathrm{Acm}^{-2}=I_{i(2)}=\frac{E_{N}-E_{A(2)}}{R_{i(2)}}=1.0334 \mathrm{Acm}^{-2}
$$

These correspond to the hydrogen consumption rates. Note also that the electronic current is given by

$$
I_{e(2)}=-\frac{E_{A(2)}}{R_{e(2)}}=-0.4622 \mathrm{Acm}^{-2}
$$

Thus,

$$
I_{L(2)}=I_{i(2)}+I_{e(2)}=0.5712 \mathrm{Acm}^{-2}
$$


Note that the ionic and the electronic currents are anti-parallel [1]. The joule heating rates in the two cells are

$$
\frac{\left(E_{N}-E_{A(1)}\right)^{2}}{R_{i(1)}}=0.534 \mathrm{Wcm}^{-2}=\frac{\left(E_{N}-E_{A(2)}\right)^{2}}{R_{i(2)}}+\frac{E_{A(2)}^{2}}{R_{e(2)}}=0.534 \mathrm{Wcm}^{-2}
$$

The power generation in the two cells is given by

$$
E_{A(1)} I_{L(1)}=0.3833 \times 1.0334=0.3961 \mathrm{Wcm}^{-2}=E_{A(2)} I_{L(2)}=0.6933 \times 0.5712=0.3961 \mathrm{Wcm}
$$

The preceding shows that both cells, one made of a purely ion conducting electrolyte (e.g. YSZ) and the other made of an MIEC electrolyte with significant electronic conductivity (such as YSZ doped with ceria, cell ionic transference number of $\sim 0.88$ ) can be operated in SOEC/SOFC modes such that they consume/generate the same amount of electricity, generate the same amount of joule heat, and generate/consume the same amount of hydrogen. Thus, insofar as the performance characteristics are concerned both cells behave identically (when operated at the respective sets of conditions). Since the fuel gas mixture contained $70 \% \mathrm{H}_{2} \mathrm{O}$ and $30 \% \mathrm{H}_{2}$, the performance is better as an SOEC than as an SOFC.

The Crossover Point: In the preceding calculations, the objective was to determine the operating conditions in both SOEC and SOFC modes under which purely ionic conducting electrolyte cells 
(e.g. YSZ) and MIEC cells exhibit identical performance characteristics. It is clear that the voltage vs. current plots for the two cells intersect in the SOFC regime. The following is an analysis of the crossover (intersection) point.

At the crossover point, we have

$$
E_{A(1)}=E_{A(2)}=E_{A}
$$

That is, both cells operate at the same measured voltage, $E_{A}$. Also, at the crossover point

$$
I_{L(1)}=\frac{E_{N}-E_{A}}{R_{i(1)}}=I_{L(2)}=\frac{E_{N}-E_{A}}{R_{i(2)}}-\frac{E_{A}}{R_{e(2)}}=I_{L}
$$

That is, both cells operate at the same externally measured current, $I_{L}$. From equation (46) we obtain

$$
E_{A}=\frac{\left(R_{i(1)}-R_{i(2)}\right) R_{e(2)} E_{N}}{\left(R_{i(1)} R_{i(2)}+R_{i(1)} R_{e(2)}-R_{i(2)} R_{e(2)}\right)}
$$

Substituting for $E_{A}$ from equation (47) into equation (46), the current is given by

$$
I_{L}=\frac{R_{i(2)} E_{N}}{\left(R_{i(1)} R_{i(2)}+R_{i(1)} R_{e(2)}-R_{i(2)} R_{e(2)}\right)}
$$


For the preceding numerical illustration, the corresponding values are $E_{A}=0.7364 \mathrm{~V}$ and $I_{L}=0.3273 \mathrm{Acm}^{-2}$. Note that although the two cells exhibit the same performance (power) at the crossover point, their performance characteristics are different since they consume hydrogen at different rates and generate joule heating at different rates. Figure 4 shows voltage vs. current plots for the two cells over both the SOEC and the SOFC regimes, including the crossover point.

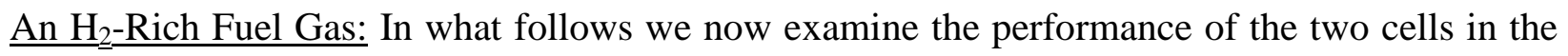
two modes. We choose $90 \% \mathrm{H}_{2}+10 \% \mathrm{H}_{2} \mathrm{O}$ as the fuel gas. The corresponding Nernst voltage is $E_{N}=1.05 \mathrm{~V}$

Using these values, we have

$$
\begin{aligned}
& a=\left(R_{i(2)}-R_{i(1)}\right) R_{e(2)}+R_{i(2)}^{2}=-0.41 \Omega^{2} \mathrm{~cm}^{4} \\
& b=2 E_{N} R_{e(2)}\left(R_{i(1)}-R_{i(2)}\right)=0.945 \mathrm{~V} \Omega^{2} \mathrm{~cm}^{4}
\end{aligned}
$$

and

$$
c=E_{N}^{2} R_{e(2)}\left(R_{i(2)}-R_{i(1)}\right)=-0.4961 \mathrm{~V}^{2} \Omega^{2} \mathrm{~cm}^{4}
$$

Solution to equation (20) is

$$
E_{A(2)}=\frac{-b \pm \sqrt{b^{2}-4 a c}}{2 a}
$$


The two values of $E_{A(2)}$ are

$$
E_{A(2)}=0.8088 \mathrm{~V} \text { and } E_{A(2)}=1.496 \mathrm{~V}
$$

In the SOFC mode, we must have $E_{A(2)}<E_{N}$. Thus, we choose $E_{A(2)}=0.8088 \mathrm{~V}$. The corresponding voltage for cell 1 is given by

$$
E_{A(1)}=E_{N}+\left(E_{A(2)}-E_{N}\right) \frac{R_{i(1)}}{R_{i(2)}}=0.4471 \mathrm{~V}
$$

Note that the ionic current densities are

$$
I_{i(1)}=\frac{E_{N}-E_{A(1)}}{R_{i(1)}}=1.2058 \mathrm{Acm}^{-2}=I_{i(2)}=\frac{E_{N}-E_{A(2)}}{R_{i(2)}}=1.206 \mathrm{Acm}^{-2}
$$

which correspond to the hydrogen consumption rates. The positive sign means the ionic currents are in the opposite direction to that in the SOEC mode. The electronic current for cell 2 is

$$
I_{e(2)}=-\frac{E_{A(2)}}{R_{e(2)}}=-0.5392 \mathrm{Acm}^{-2}
$$

Note that the ionic and the electronic currents are anti-parallel [1]. The joule heating for the two cells is given by 


$$
\frac{\left(E_{N}-E_{A(1)}\right)^{2}}{R_{i(1)}}=0.727 \mathrm{Wcm}^{-2}=\frac{\left(E_{N}-E_{A(2)}\right)^{2}}{R_{i(2)}}+\frac{E_{A(2)}^{2}}{R_{e(2)}}=0.727 \mathrm{Wcm}^{-2}
$$

The load current for cell 2 is

$$
I_{L(2)}=I_{i(2)}+I_{e(2)}=0.6667 \mathrm{Acm}^{-2}
$$

Finally, the electrical power generation rates for the two cells are identical and are given by

$$
E_{A(1)} I_{L(1)}=0.4471 \times 1.2059=0.5392 \mathrm{Wcm}^{-2}=E_{A(2)} I_{L(2)}=0.7703 \times 0.6351=0.5392 \mathrm{Wcm}^{-2}
$$

Note that the above are the estimates of electrical power generation by the cells. These are positive, which means the cells deliver power, since they are operating as SOFC. Thus, both cells generate the same electrical power, given by $0.5392 \mathrm{Wcm}^{-2}$.

Now let us choose $E_{A(2)}=1.496 \mathrm{~V}$. This is the SOEC mode. The corresponding $E_{A(1)}$ is given by

$$
E_{A(1)}=E_{N}+\left(E_{A(2)}-E_{N}\right) \frac{R_{i(1)}}{R_{i(2)}}=2.165 \mathrm{~V}
$$

Note that the ionic current densities are given by 


$$
I_{i(1)}=\frac{E_{N}-E_{A(1)}}{R_{i(1)}}=-2.23 \mathrm{Acm}^{-2}=I_{i(2)}=\frac{E_{N}-E_{A(2)}}{R_{i(2)}}=-2.23 \mathrm{Acm}^{-2}
$$

which correspond to the hydrogen generation rates. The electronic current is given by

$$
I_{e(2)}=-\frac{E_{A(2)}}{R_{e(2)}}=-0.9973 \mathrm{Acm}^{-2}
$$

Thus, the load current is

$$
I_{L(2)}=I_{i(2)}+I_{e(2)}=-3.2273 \mathrm{Acm}^{-2}
$$

Note that the ionic and the electronic currents are parallel [1]. The joule heating rates for the two cells are given by

$$
\frac{\left(E_{N}-E_{A(1)}\right)^{2}}{R_{i(1)}}=2.4865 \mathrm{Wcm}^{-2}=\frac{\left(E_{N}-E_{A(2)}\right)^{2}}{R_{i(2)}}+\frac{E_{A(2)}^{2}}{R_{e(2)}}=2.4865 \mathrm{Wcm}^{-2}
$$

Finally, the electrical power generation rates are given by

$$
E_{A(1)} I_{L(1)}=2.165 \times(-2.23)=-4.828 \mathrm{Wcm}^{-2}=E_{A(2)} I_{L(2)}=1.496 \times(-3.2273)=-4.828 \mathrm{Wcm}^{-2}
$$


Negative values means these are the power consumption rates. Note that both cells behave identically insofar as the performance characteristics are concerned. The crossover point for this case is given by $E_{A}=0.8591 \mathrm{~V}$ and $I_{L}=0.3818 \mathrm{Acm}^{-2}$. In the above case, the fuel gas is rich in $\mathrm{H}_{2}$ but with relatively low $\mathrm{H}_{2} \mathrm{O}$ content (10\%). As such, the performance characteristics are not good in the SOEC mode. It is understood that one would not operate with an $\mathrm{H}_{2}$-rich fuel electrode gas if the cells are to be operated in the SOEC mode. The preceding shows that MIEC cells can be designed to operate as efficiently as those made of a purely ionic conducting electrolyte in both modes under various gas compositions. Thus, they can be operated in an $\mathrm{H}_{2} \mathrm{O}-$ rich fuel gas in the SOEC mode and in an $\mathrm{H}_{2}$-rich fuel gas in the SOFC mode.

General Equations for Power, Hydrogen Consumption Rate and Joule Heating for an MIEC Solid Oxide Fuel Cell (SOFC):

In what follows, general equations for an MIEC cell are given. As before, the ionic resistance is given by $R_{i}$ and the electronic resistance is given by $R_{e}$. Figure 2 shows the simplified equivalent circuit.

The voltage vs. current plot is given by

$$
E_{A}=E_{N}\left(\frac{R_{e}}{R_{i}+R_{e}}\right)-I_{L}\left(\frac{R_{e} R_{i}}{R_{i}+R_{e}}\right)
$$


At open circuit,

$$
E_{A}=E_{N}\left(\frac{R_{e}}{R_{i}+R_{e}}\right)=E_{N} t_{i}
$$

At short circuit, the measured current is given by

$$
I_{s}=\frac{E_{N}}{R_{i}}
$$

The electrical power is given by

$$
P=E_{A} I_{L}=E_{N} I_{L}\left(\frac{R_{e}}{R_{i}+R_{e}}\right)-I_{L}^{2}\left(\frac{R_{e} R_{i}}{R_{i}+R_{e}}\right)
$$

Note that $P=0$ for $I_{L}=0$ (open circuit), $P=0$ for $I_{L}=I_{S}=\frac{E_{N}}{R_{i}}$ (short circuit), and $P>0$ for $0 \leq I_{L} \leq I_{S}$. This is the SOFC mode. For $I_{L}<0$, the $P<0$. This is the SOEC mode, in which electrical energy is supplied to the cell.

The joule heating rate is given by

$$
\dot{Q}=I_{i}^{2} R_{i}+I_{e}^{2} R_{e}=\frac{\left(E_{N}-E_{A}\right)^{2}}{R_{i}}+\frac{E_{A}^{2}}{R_{e}}
$$


Substituting for $E_{A}$ in terms of $E_{N}, I_{L}$ and the various resistances, the joule heating rate is given by

$$
\dot{Q}=\frac{E_{N}^{2}}{\left(R_{i}+R_{e}\right)}+\left(\frac{R_{i} R_{e}}{R_{i}+R_{e}}\right) I_{L}^{2}
$$

Note that the $\dot{Q}$ is always positive as to be expected. Its lowest value occurs at open circuit when $I_{L}=0$, which is given by

$$
\dot{Q}_{O C V}=\frac{E_{N}^{2}}{\left(R_{i}+R_{e}\right)}
$$

The ionic current is given by

$$
I_{i}=\frac{E_{N}}{\left(R_{i}+R_{e}\right)}+\left(\frac{R_{e}}{R_{i}+R_{e}}\right) I_{L}
$$

The ionic current is a measure of the hydrogen consumption rate (when $I_{i}>0$, SOFC mode) or hydrogen generation rate (when $I_{i}<0$, SOEC mode). We first consider the SOFC mode. We will write

$$
I_{H}=I_{i}=\frac{E_{N}}{\left(R_{i}+R_{e}\right)}+\left(\frac{R_{e}}{R_{i}+R_{e}}\right) I_{L}
$$


where $I_{H}$ in $\mathrm{Acm}^{-2}$ is a measure of the hydrogen consumption rate. In what follows, we will fix the hydrogen consumption rate at some value $I_{H}^{o}$, corresponding to a purely ionic conducting electrolyte cell.

If the cell is made of a purely ionic conductor with the ionic resistance as $R_{i}^{o}$, then

$$
I_{H}=I_{i}=\frac{E_{N}}{\left(R_{i}^{o}+R_{e}^{o}\right)}+\left(\frac{R_{e}^{o}}{R_{i}^{o}+R_{e}^{o}}\right) I_{L}=I_{L}=I_{L}^{o}=I_{H}^{o}
$$

in which $R_{e}^{o} \rightarrow \infty$.

We will also fix power density at some value $P^{o}$, corresponding to the purely ionic conducting electrolyte cell.

The power density is given by

$$
P=E_{A} I_{L}=E_{N} I_{L}\left(\frac{R_{e}}{R_{i}+R_{e}}\right)-I_{L}^{2}\left(\frac{R_{e} R_{i}}{R_{i}+R_{e}}\right)=\left(E_{N}-I_{L}^{o} R_{i}^{o}\right) I_{L}^{o}=P^{o}
$$

We now consider another cell made of an MIEC electrolyte such that the ionic and the electronic resistances respectively are $R_{i}$ and $R_{e}$. We want to select values of $R_{i}$ and $R_{e}$ such that the MIEC cell consumes hydrogen at the same rate as the purely ion conducting cell and also delivers the same power as the purely ion conducting cell. In what follows, we first choose a 
value of $R_{i}$, and then determine the corresponding value of $R_{e}$, such that the hydrogen consumption rate is $I_{H}^{o}$, and the power is $P^{o}$, which are those corresponding to the purely ionic conducting electrolyte cell.

From equations (73), (74) and (75), we can write an equation in $R_{e}$ as a function of the other parameters including $R_{i}$ in which $I_{L}$ is written in terms of $I_{H}^{o}$ and the power is given by $P^{o}$. The equation is

$$
\left(P^{o}-E_{N} I_{H}^{o}+\left(I_{H}^{o}\right)^{2} R_{i}\right) R_{e}^{2}+\left(P^{o} R_{i}+E_{N}^{2}+2\left(I_{H}^{o}\right)^{2} R_{i}^{2}-3 I_{H}^{o} E_{N} R_{i}\right) R_{e}+\left(\left(I_{H}^{o}\right)^{2} R_{i}^{3}+E_{N}^{2} R_{i}-2 I_{H}^{o} E_{N} R_{i}^{2}\right)=0
$$

Note that this is a quadratic equation in $R_{e}$, namely

$$
a^{\prime} R_{e}^{2}+b^{\prime} R_{e}+c^{\prime}=0
$$

where

$$
\begin{aligned}
& a^{\prime}=\left(P^{o}-E_{N} I_{H}^{o}+\left(I_{H}^{o}\right)^{2} R_{i}\right) \\
& b^{\prime}=\left(P^{o} R_{i}+E_{N}^{2}+2\left(I_{H}^{o}\right)^{2} R_{i}^{2}-3 I_{H}^{o} E_{N} R_{i}\right)
\end{aligned}
$$


and

$$
c^{\prime}=\left(\left(I_{H}^{o}\right)^{2} R_{i}^{3}+E_{N}^{2} R_{i}-2 I_{H}^{o} E_{N} R_{i}^{2}\right)
$$

The solution is given by

$$
R_{e}=\frac{-b^{\prime} \pm \sqrt{b^{\prime 2}-4 a^{\prime} c^{\prime}}}{2 a^{\prime}}
$$

Only the positive roots will be realistic. The preceding shows that, for a given $I_{H}^{o}$ (hydrogen consumption rate) and for a given $P^{o}$ (power density), that is for a given set of (arbitrarily selected) performance parameters for a purely ion conducting cell, if one arbitrarily chooses $R_{i}$ for an MIEC cell, then from the preceding equations, one can determine what should be the value of $R_{e}$ for the MIEC cell that ensures identical performance characteristics as the purely ionic conducting electrolyte cell.

Numerical Illustration: We first consider a purely ion conducting electrolyte cell. The operating temperature is selected as $800^{\circ} \mathrm{C}$. We assume its area specific resistance $R_{i}^{o}=0.5 \Omega \mathrm{cm}^{2}$. We select the operating current density as $I_{L}^{o}=1.0 \mathrm{Acm}^{-2}=I_{H}^{o}$. Also, we choose $90 \% \mathrm{H}_{2}+10 \% \mathrm{H}_{2} \mathrm{O}$ as the fuel gas. The corresponding Nernst voltage is $E_{N}=1.05 \mathrm{~V}$. The corresponding cell voltage is $E_{A}^{o}=0.55 \mathrm{~V}$ and the corresponding power density is $P^{o}=0.55 \mathrm{Wcm}^{-2}$. 
We now choose an MIEC cell. We assume that the ionic area specific resistance of the MIEC cell is $R_{i}=0.25 \Omega \mathrm{cm}^{2}$. The objective now is to calculate what should be the corresponding electronic area specific resistance, $R_{e}$, which ensures that the cell at its operating conditions gives a power of $0.55 \mathrm{Wcm}^{-2}$ and consumes hydrogen at a rate corresponding to $1 \mathrm{Acm}^{-2}$. The coefficients are

$$
\begin{aligned}
& a^{\prime}=\left(P^{o}-E_{N} I_{H}^{o}+\left(I_{H}^{o}\right)^{2} R_{i}\right)=-0.25 \mathrm{Wcm}^{-2} \\
& b^{\prime}=\left(P^{o} R_{i}+E_{N}^{2}+2\left(I_{H}^{o}\right)^{2} R_{i}^{2}-3 I_{H}^{o} E_{N} R_{i}\right)=0.5775 \mathrm{~W} \Omega \\
& c^{\prime}=\left(\left(I_{H}^{o}\right)^{2} R_{i}^{3}+E_{N}^{2} R_{i}-2 I_{H}^{o} E_{N} R_{i}^{2}\right)=0.16 \mathrm{~W} \Omega^{2} \mathrm{~cm}^{2}
\end{aligned}
$$

Solution to the quadratic equation (76) gives $R_{e}=2.56 \Omega \mathrm{cm}^{2}$. The corresponding load current is $I_{L}=0.6875 \mathrm{Acm}^{-2}$. The corresponding cell voltage is $E_{A}=0.8 \mathrm{~V}$. This means the electronic current is $I_{e}=-\frac{E_{A}}{R_{e}}=-\frac{0.8}{2.56}=-0.3125 \mathrm{Acm}^{-2}$. Note that the cell ionic transference number is $t_{i}=\frac{R_{e}}{R_{i}+R_{e}}=0.911$

General Equations for Power Input, Hydrogen Generation Rate and Joule Heating for an MIEC $\underline{\text { Solid Oxide Electrolyzer Cell (SOEC): }}$

The same equations are also applicable in the SOEC mode. In the present case note that 


$$
I_{H}=I_{i}=\frac{E_{N}}{\left(R_{i}+R_{e}\right)}+\left(\frac{R_{e}}{R_{i}+R_{e}}\right) I_{L}
$$

is the hydrogen generation rate. In this case, the $I_{L}<0$ and also $I_{H}=I_{i}<0$. Similarly, the power $P<0$, and represents energy input into the cell.

Numerical Illustration: We choose $70 \% \mathrm{H}_{2} \mathrm{O}+30 \% \mathrm{H}_{2}$ as the fuel gas. The corresponding Nernst voltage is $E_{N}=0.9 \mathrm{~V}$. Once again we first consider an SOEC made of a purely ionic conducting electrolyte. We will assume hydrogen generation rate corresponding to $-1 \mathrm{Acm}^{-2}$; that is $I_{L}^{o}=I_{H}^{o}=-1 \mathrm{Acm}^{-2}$. Also, we will assume $R_{i}^{o}=0.5 \Omega \mathrm{cm}^{2}$. The corresponding cell voltage is $E_{A}^{o}=1.4 \mathrm{~V}$. Also, the corresponding power input is $P^{o}=-1.4 \mathrm{Wcm}^{-2}$. Let us again assume that for the MIEC cell, $R_{i}=0.25 \Omega \mathrm{cm}^{2}$. Now the coefficients are

$$
\begin{aligned}
& a^{\prime}=\left(P^{o}-E_{N} I_{H}^{o}+\left(I_{H}^{o}\right)^{2} R_{i}\right)=-0.75 \mathrm{Wcm}^{-2} \\
& b^{\prime}=\left(P^{o} R_{i}+E_{N}^{2}+2\left(I_{H}^{o}\right)^{2} R_{i}^{2}-3 I_{H}^{o} E_{N} R_{i}\right)=1.26 \mathrm{~W} \Omega \\
& c^{\prime}=\left(\left(I_{H}^{o}\right)^{2} R_{i}^{3}+E_{N}^{2} R_{i}-2 I_{H}^{o} E_{N} R_{i}^{2}\right)=0.2901 \mathrm{~W} \Omega^{2} \mathrm{~cm}^{2}
\end{aligned}
$$


Solution to the quadratic equation (76) gives $R_{e}=1.8852 \Omega \mathrm{cm}^{2}$. The corresponding load current is $I_{L}=-1.61 \mathrm{Acm}^{-2}$. The corresponding cell voltage is $E_{A}=1.15 \mathrm{~V}$. This means the electronic current is $I_{e}=-\frac{E_{A}}{R_{e}}=-\frac{1.15}{1.8852}=-0.61 \mathrm{Acm}^{-2}$. Note that the cell ionic transference number is $t_{i}=\frac{R_{e}}{R_{i}+R_{e}}=0.8829$.

The preceding shows an approach to estimating a given set of cell parameters (ionic and electronic resistances) to ensure that MIEC electrolyte cells exhibit the same performance characteristics as purely ion conducting electrolyte cells. In what follows, we describe some experimental results on 5 cell stacks of purely ion conducting electrolyte cells and MIEC cells.

\section{Experimental Results and Analysis}

Experimental Results on SOEC and SOFC Stacks made of Purely Ion Conducting Electrolytes and MIEC Electrolytes: Experimental studies were conducted on 5 cell stacks. The cells were Ni + YSZ fuel electrode-supported with a thin (thickness $~ 10$ micron) electrolyte and an active area of $100 \mathrm{~cm}^{2}$. The oxygen electrode was a composite of a perovskite-based material mixed with YSZ. One set of cells was made with 8YSZ as the electrolyte. The other set of cells was made using 8YSZ doped with 8 mol. $\% \mathrm{CeO}_{2}$ (8CYSZ). The objective of doping with $\mathrm{CeO}_{2}$ was to generate mixed ionic electronic conductivity (MIEC) in the electrolyte. The cell active area was $100 \mathrm{~cm}^{2}$. Five cell stacks were assembled using metallic interconnects having built-in flow-fields 
for fuel and oxidant. The cells were sealed at the edges using glass-mica seals. Stacks were compression-loaded using a fixture. All tests were conducted at $800^{\circ} \mathrm{C}$.

Tests were conducted in both SOEC and SOFC modes. Gaseous mixtures of $\mathrm{H}_{2}$ and $\mathrm{H}_{2} \mathrm{O}$ containing a wide range of partial pressures were used as the fuel electrode gas for conducting the tests. For the SOEC tests, the gaseous mixture was typically $\mathrm{H}_{2} \mathrm{O}$ rich. For the SOFC tests, the gaseous mixture was typically $\mathrm{H}_{2}$ rich.

An SOEC-SOFC Five Cell Stack with 8YSZ Electrolyte: Figure 5 shows the experimentally measured performance curves for an SOEC-SOFC stack with 8YSZ cells. The OCV/cell for $90 \%$ $\mathrm{H}_{2} \mathrm{O}+10 \% \mathrm{H}_{2}$ was $0.84 \mathrm{~V}$, while that calculated from thermodynamic tables is $0.8324 \mathrm{~V}$. The experimentally measured OCV/cell for $50 \% \mathrm{H}_{2} \mathrm{O}+50 \% \mathrm{H}_{2}$ was $0.94 \mathrm{~V}$, while that calculated is $0.934 \mathrm{~V}$. The experimentally measured OCV/cell with $\sim 100 \% \mathrm{H}_{2}$ (with a small $\mathrm{H}_{2} \mathrm{O}$ content) was about $1.08 \mathrm{~V}$. This corresponds to $\sim 96 \% \mathrm{H}_{2}+4 \% \mathrm{H}_{2} \mathrm{O}$. These observations also are consistent with YSZ being an essentially purely ionic conductor. Green lines drawn in the figure indicate the approximate voltage vs. current traces. These traces were used for performance calculations and analysis. For SOEC tests, the data used for calculations (green line in the figure) correspond to a fuel gas composition of $70 \% \mathrm{H}_{2} \mathrm{O}+30 \% \mathrm{H}_{2}$. The corresponding $\mathrm{OCV}$ (which is assumed to be the Nernst voltage) is $0.86 \mathrm{~V}$. For the SOFC tests, the green line corresponds to $\sim 100 \% \mathrm{H}_{2}$ (with a small $\mathrm{H}_{2} \mathrm{O}$ content). There is considerable curvature in the voltage vs. current trace, and the green line drawn is an approximation. 
An SOEC-SOFC Stack with 8CYSZ Electrolyte: Figure 6 shows performance curves for a stack with cells made using 8 CYSZ electrolyte. For nearly $100 \% \mathrm{H}_{2}$ as the fuel, the measured OCV was $0.98 \mathrm{~V}$. Assuming that the $\mathrm{H}_{2} \mathrm{O}$ content in nearly $100 \% \mathrm{H}_{2}$ gas was $\sim 4 \%$, the transference number for the cell is estimated to be $\sim 0.9$. For a gaseous mixture containing $50 \% \mathrm{H}_{2}+50 \%$ $\mathrm{H}_{2} \mathrm{O}$, the OCV/cell is about $0.9 \mathrm{~V}$. This corresponds to an ionic transference number between about 0.9 and 0.95 . Finally, for $80 \% \mathrm{H}_{2} \mathrm{O}+20 \% \mathrm{H}_{2}$, the $\mathrm{OCV} /$ cell (there is some deviation from a straight line behavior) is $\sim 0.76 \mathrm{~V}$. This again corresponds to a transference number of about 0.9. Green lines drawn in the figure indicate the approximate voltage vs. current traces (corresponding to $\sim 100 \% \mathrm{H}_{2}$ for SOFC and $70 \% \mathrm{H}_{2} \mathrm{O}+30 \% \mathrm{H}_{2}$ for SOEC). These traces are used for performance calculations. For SOEC tests, data used for calculations correspond to $70 \%$ $\mathrm{H}_{2} \mathrm{O}+30 \% \mathrm{H}_{2}$. For SOFC tests, data used for the calculations correspond to $\sim 100 \% \mathrm{H}_{2}$.

Figure 5 and Figure 6 also show that the cell area specific resistance of the ceria-doped (8CYSZ) cells is much lower than that of the purely ion conducting 8YSZ electrolyte cells. The ionic conductivity of ceria-doped YSZ is not expected to be far different from that of YSZ. Thus, the much lower area specific resistance of $8 \mathrm{CYSZ}$ is attributed to the lower polarization resistance in the $8 \mathrm{CYSZ}$ cells. The reason for very high cell resistance for the $8 \mathrm{YSZ}$ cells is the poor quality of the oxygen electrode, which was not optimized for use as the oxygen electrode for the 8YSZ cells, especially in the SOEC mode. For most of the subsequent work different oxygen electrodes are used in our work.

Analysis of Stack Tests: The performance curves show that the area specific resistance of the 8YSZ cells (Figure 5) is higher in the SOEC mode than in the SOFC mode. In 8CYSZ cells 
(Figure 6), the area specific resistance is about the same in both modes. Green lines in the figures are drawn to show approximate values of the resistance. The negative of the slope gives the stack resistance, namely

$$
R_{\text {stack }}=\mid \text { Slope } \mid
$$

The repeat unit area specific resistance is given by

$$
R_{\text {cell }}=\frac{R_{\text {stack }}}{5} \times 100
$$

This value includes interconnect and possible contact resistances. In what follows, it is assumed that they are small, and thus neglected here.

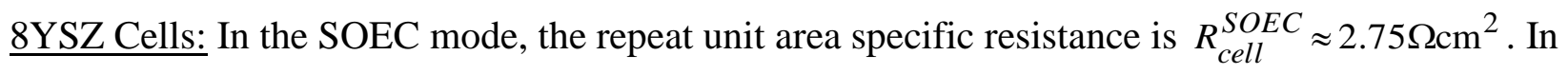
the SOFC mode, the corresponding value is $R_{\text {cell }}^{S O F C} \approx 1.72 \Omega \mathrm{cm}^{2}$. As stated earlier, the oxygen electrode used with 8YSZ cell in this work was of poor quality. Because of the substantial nonlinearity in voltage vs. current data in the SOFC mode, the value of $R_{\text {cell }}^{S O F C} \approx 1.72 \Omega \mathrm{cm}^{2}$ is only an approximate estimate. As mentioned earlier, the overall cell ionic resistance, $R_{i}$, includes activation polarization resistances at the two electrodes. The electrolyte resistance is not expected to be far different in the two modes. Thus, the higher value of the cell resistance in the SOEC mode is attributed to relatively high polarization resistance of the oxygen electrode with $8 \mathrm{YSZ}$ as the electrolyte. In the SOFC mode, the fuel electrode is exposed to a predominantly 
hydrogen containing atmosphere. It is well known that $\mathrm{Ni}$ is an excellent electrocatalyst for anode reactions in SOFC. In the SOEC mode, the fuel electrode is exposed to a higher $\mathrm{H}_{2} \mathrm{O}$ content and a lower $\mathrm{H}_{2}$ content compared to the SOFC mode. In addition, the polarization resistances at a given electrode can be different in the two modes. The higher overall cell resistance in the SOEC mode seems to suggest that with $8 \mathrm{YSZ}$ as the electrolyte, $\mathrm{Ni}+\mathrm{YSZ}$ as the fuel electrode and oxide perovskite + YSZ as the oxygen electrode the polarization resistance is higher in the SOEC mode than in the SOFC mode.

8CYSZ Cells: The magnitudes of the slopes to the green lines drawn in Figure 6 again give the stack resistance. In 8CYSZ cells, the measured cell resistance in the SOEC mode is $R_{\text {cell }}^{S O E C} \approx 0.5 \Omega \mathrm{cm}^{2}$ and that in the SOFC mode is $R_{\text {cell }}^{S O F C} \approx 0.48 \Omega \mathrm{cm}^{2}$, that is about the same in both modes. It is also important to note that the cell resistance is considerably lower in 8CYSZ MIEC cells compared to $8 \mathrm{YSZ}$ cells. The ionic transference number is about $\sim 0.9$. Also, the ionic conductivity of $8 \mathrm{CYSZ}$ is about the same as that of $8 \mathrm{YSZ}$. This means much of the difference in cell resistances between $8 \mathrm{YSZ}$ and $8 \mathrm{CYSZ}$ cells is related to differences in polarization resistances (primarily the oxygen electrode), and not to any differences in the ionic contribution of the electrolyte in the two cases.

SOEC Mode: We now compare the results of $8 \mathrm{YSZ}$ and $8 \mathrm{CYSZ}$ cells (repeat units) in the SOEC mode. For the $8 \mathrm{YSZ}$ cell, $R_{\text {cell }}^{S O E C}=R_{i(1)} \approx 2.75 \Omega \mathrm{cm}^{2}$. For the $8 \mathrm{CYSZ}$ cell, $R_{\text {cell }}^{S O E C} \approx 0.5 \Omega \mathrm{cm}^{2}$. The corresponding ionic transference number is $t_{i} \approx 0.9$. From these two measurements, we estimate the ionic and the electronic area specific resistances of the 8CYSZ cell as 
$R_{i(2)} \approx 0.556 \Omega \mathrm{cm}^{2}$ and $R_{e(2)} \approx 5.0 \Omega \mathrm{cm}^{2}$, respectively. We will choose fuel gas as $70 \% \mathrm{H}_{2} \mathrm{O}-$ $30 \% \mathrm{H}_{2}$. The corresponding Nernst voltage is $E_{N}=0.9 \mathrm{~V}$.

Thus, the coefficients of the quadratic equation (20) are

$$
\begin{aligned}
& a=\left(R_{i(2)}-R_{i(1)}\right) R_{e(2)}+R_{i(2)}^{2}=-10.661 \Omega^{2} \mathrm{~cm}^{4} \\
& b=2 E_{N} R_{e(2)}\left(R_{i(1)}-R_{i(2)}\right)=19.746 \mathrm{~V} \Omega^{2} \mathrm{~cm}^{4}
\end{aligned}
$$

and

$$
c=E_{N}^{2} R_{e(2)}\left(R_{i(2)}-R_{i(1)}\right)=-8.8857 \mathrm{~V}^{2} \Omega^{2} \mathrm{~cm}^{4}
$$

The solution is given by

$$
E_{A(2)}=\frac{-b \pm \sqrt{b^{2}-4 a c}}{2 a}
$$

There are two values of $E_{A(2)} ; E_{A(2)}=1.0815 \mathrm{~V}$ and $E_{A(2)}=0.7707 \mathrm{~V}$. In the SOEC mode, the cell voltage must be greater than the open circuit voltage. Thus, we select $E_{A(2)}=1.0815 \mathrm{~V}$.

The corresponding $E_{A(1)}$ is given by 


$$
E_{A(1)}=E_{N}+\left(E_{A(2)}-E_{N}\right) \frac{R_{i(1)}}{R_{i(2)}}=1.7977 \mathrm{~V}
$$

That is, to ensure identical performance, the 8YSZ cells should be operated at $E_{A(1)}=1.7977 \mathrm{~V}$ and 8CYSZ cells should be operated at $E_{A(2)}=1.0815 \mathrm{~V}$.

The corresponding ionic currents are

$$
I_{i(1)}=\frac{E_{N}-E_{A(1)}}{R_{i(1)}}=-0.3264 \mathrm{Acm}^{-2}=I_{i(2)}=\frac{E_{N}-E_{A(2)}}{R_{i(2)}}=-0.3264 \mathrm{Acm}^{-2}
$$

This is essentially the hydrogen generation rate.

The electronic current in the $8 \mathrm{CYSZ}$ cell is

$$
I_{e(2)}=-\frac{E_{A(2)}}{R_{e(2)}}=-0.2163 \mathrm{Acm}^{-2}
$$

Thus, for these values of the operating parameters for the 8CYSZ cells, the electronic current is substantial. The net current density through the $8 \mathrm{CYSZ}$ cell is

$$
I_{L(2)}=\frac{E_{N}-E_{A(2)}}{R_{i(2)}}-\frac{E_{A(2)}}{R_{e(2)}}=-0.5427 \mathrm{Acm}^{-2}
$$


The electrical energy generation rates, if the $8 \mathrm{YSZ}$ and $8 \mathrm{CYSZ}$ cells are to be operated at the calculated conditions, are

$$
E_{A(1)} I_{L(1)}=1.7977 \times(-0.3264)=-0.5868 \mathrm{Wcm}^{-2}=E_{A(2)} I_{L(2)}=1.0815 \times(-0.5427)=-0.5868 \mathrm{Wcm}^{-2}
$$

The electrical energy generation rate is negative, which means electrical energy is absorbed to generate hydrogen and joule heating.

A comparison of Figure 5 and Figure 6 shows that neither of the cells were operated under these conditions. However, the extrapolated values are consistent with the calculations. This is discussed in what follows.

The estimated $E_{A(2)}$ is $1.0815 \mathrm{~V}$. Thus, for the 8CYSZ five cell stack, the stack voltage should have been $5.407 \mathrm{~V}$ and the net current should have been -54 A. But the 8CYSZ stack in the SOEC mode was operated only at a maximum voltage of $\sim 4.65 \mathrm{~V}$ at a current of $\sim-14 \mathrm{~A}$. If it had been operated at $5.4 \mathrm{~V}$, the corresponding current (by extrapolating the green line) would have been -46 A, in reasonable agreement with calculations. Figure 6 shows the corresponding extrapolation (green dot on the extrapolated line, SOEC side).

For the $8 \mathrm{YSZ}$ stack, the calculated $E_{A(1)}$ is about $\sim 1.8 \mathrm{~V}$. The corresponding stack voltage should be $\sim 9 \mathrm{~V}$. But the stack was only operated at $\sim 6.3 \mathrm{~V}$ and the corresponding current was only -14 A.. The estimated stack current for a stack voltage of $9 \mathrm{~V}$ is estimated to be $\sim-33 \mathrm{~A}$. If 
the stack had been operated at $9 \mathrm{~V}$, the extrapolation shows that the stack current would have been -32 A, in excellent agreement with calculations. Figure 5 shows the corresponding extrapolation (green dot on the extrapolated line, SOEC side).

SOFC Mode: We now compare the results of the 8YSZ and 8CYSZ cells in the SOFC mode. We select $\sim 100 \% \mathrm{H}_{2}$ as the fuel gas. The corresponding Nernst voltage is $E_{N}=1.08 \mathrm{~V}$. For the $8 \mathrm{YSZ}$ cells, $R_{\text {cell }}^{S O F C}=R_{i(1)} \approx 1.72 \Omega \mathrm{cm}^{2}$. For the $8 \mathrm{CYSZ}$ cells, $R_{\text {cell }}^{S O F C} \approx 0.48 \Omega \mathrm{cm}^{2}$. The corresponding ionic transference number is $t_{i} \approx 0.9$. From these two measurements, we estimate the ionic and the electronic area specific resistances of the $8 \mathrm{CYSZ}$ cell as $R_{i(2)} \approx 0.533 \Omega \mathrm{cm}^{2}$ and $R_{e(2)} \approx 4.797 \Omega \mathrm{cm}^{2}$

Thus, the coefficients of the quadratic equation (20) are

$$
\begin{aligned}
& a=\left(R_{i(2)}-R_{i(1)}\right) R_{e(2)}+R_{i(2)}^{2}=-5.41 \Omega^{2} \mathrm{~cm}^{4} \\
& b=2 E_{N} R_{e(2)}\left(R_{i(1)}-R_{i(2)}\right)=12.299 \mathrm{~V} \Omega^{2} \mathrm{~cm}^{4}
\end{aligned}
$$

and

$$
c=E_{N}^{2} R_{e(2)}\left(R_{i(2)}-R_{i(1)}\right)=-6.641 \mathrm{~V}^{2} \Omega^{2} \mathrm{~cm}^{4}
$$

Now, the solution is given by 


$$
E_{A(2)}=\frac{-b \pm \sqrt{b^{2}-4 a c}}{2 a}
$$

There are two values of $E_{A(2)} ; E_{A(2)}=1.3907 \mathrm{~V}$ and $E_{A(2)}=0.8827 \mathrm{~V}$. In the SOFC mode, the cell voltage must be lower than the open circuit voltage. Thus, we select $E_{A(2)}=0.8827 \mathrm{~V}$.

The corresponding $E_{A(1)}$ is given by

$$
E_{A(1)}=E_{N}+\left(E_{A(2)}-E_{N}\right) \frac{R_{i(1)}}{R_{i(2)}}=0.4433 \mathrm{~V}
$$

Thus, to ensure identical performance, $8 \mathrm{YSZ}$ cells should be operated at $E_{A(1)}=0.4433 \mathrm{~V}$ and $8 \mathrm{CYSZ}$ cell should be operated at $E_{A(2)}=0.8827 \mathrm{~V}$. The corresponding ionic currents are

$$
I_{i(1)}=\frac{E_{N}-E_{A(1)}}{R_{i(1)}}=0.3702 \mathrm{Acm}^{-2}=I_{i(2)}=\frac{E_{N}-E_{A(2)}}{R_{i(2)}}=0.3702 \mathrm{Acm}^{-2}
$$

This is essentially the hydrogen consumption rate.

The electronic current in the 8CYSZ cell is

$$
I_{e(2)}=-\frac{E_{A(2)}}{R_{e(2)}}=-0.184 \mathrm{Acm}^{-2}
$$


Thus, the corresponding load current is

$$
I_{L(2)}=\frac{E_{N}-E_{A(2)}}{R_{i(2)}}-\frac{E_{A(2)}}{R_{e(2)}}=0.1862 \mathrm{Acm}^{-2}
$$

The electrical energy generation rate (power) if $8 \mathrm{YSZ}$ and $8 \mathrm{CYSZ}$ cells are to be operated under the calculated conditions is given by

$$
E_{A(1)} I_{L(1)}=0.4433 \times 0.3702=0.1641 \mathrm{Wcm}^{-2}=E_{A(2)} I_{L(2)}=0.8827 \times 0.1862=0.1644 \mathrm{Wcm}^{-2}
$$

A comparison of Figure 5 and Figure 6 shows that neither of the cells were operated under these conditions. However, the extrapolated (and in one case intrapolated, green dot in Figure 6 on the SOFC side) values are consistent with the calculations. This is discussed in what follows.

The calculated $E_{A(1)}(8 \mathrm{YSZ}$ stack) is $0.44 \mathrm{~V}$. The corresponding calculated stack current is $\sim 37$ A. However, the lowest voltage the cells (repeat units) were operated at was $0.72 \mathrm{~V}$. The actual operating current was $\sim 20$ A. Extrapolation shows that if the operating voltage per cell had been $\sim 0.44 \mathrm{~V}$, the corresponding current would have been $\sim 37 \mathrm{~A}$, in excellent agreement with calculations. Figure 5 shows the corresponding extrapolation (green dot on the extrapolated line, SOFC side). 
The calculated $E_{A(2)}$ is about $0.88 \mathrm{~V}$, and the calculated current is $\sim 20 \mathrm{~A}$. An examination of Figure 6 in fact shows that for an operating cell voltage of $0.88 \mathrm{~V}$ (stack voltage of $4.4 \mathrm{~V}$ ), the current is about $20 \mathrm{~A}$, again in very good agreement with calculations. The stack was actually operated at a current as high as $30 \mathrm{~A}$. The corresponding cell voltage was $\sim 0.84 \mathrm{~V}$ (stack voltage of about $42 \mathrm{~V}$ ). That is the stack was operated past the calculated conditions.

Long Term Testing and Durability of SOEC Stacks made with 8YSZ and 8CYSZ Electrolytes: Prior theoretical and considerable experimental work has shown that degradation of cells/stacks is much more common and often severe in the SOEC mode $[10,11]$. In the SOEC mode, the often observed degradation involves delamination along the oxygen electrode/electrolyte interface [11]. This delamination is usually very clean in that it precisely occurs along the interface. In addition to delamination along the interface, numerous cracks may also appear inside the electrolyte, which are nearly parallel to the interface and in close proximity to the oxygen electrode [11]. Additional mode of degradation involves local reduction of the electrolyte near the fuel electrode/electrolyte interface [11]. This degradation manifests as the formation of voids. Theoretical analysis has shown that oxygen electrode delamination, the formation of cracks in the electrolyte and reduction of the electrolyte near the fuel electrode are all related to the fundamentals of transport and linear non-equilibrium thermodynamics [2]. Specifically, it has been shown that in the SOEC mode, the oxygen ion current and the electron current (however small) through the electrolyte are parallel to each other. Under such conditions, the chemical potential of oxygen, $\mu_{O_{2}}(x)$, inside the electrolyte need not be mathematically bounded by the values at the electrodes $[1,2]$. Thus, the $\mu_{O_{2}}(x)$ in the electrolyte close to the oxygen electrode $\left(\mu_{O_{2}}^{c}\right.$ ) can exceed the value at the oxygen electrode $\left(\mu_{O_{2}}^{I}\right)$, resulting in high internal oxygen 
pressures in the electrolyte close to the oxygen electrode leading to internal cracks and oxygen electrode delamination. Similarly, the $\mu_{O_{2}}(x)$ in the electrolyte close to the fuel electrode $\left(\mu_{O_{2}}^{a}\right)$ can decrease below the value at the fuel electrode $\left(\mu_{O_{2}}^{I I}\right)$. Under such conditions oxygen partial pressure in the electrolyte close to the fuel electrode may become much lower than the value in the fuel electrode. In such cases partial (or even total) reduction of the electrolyte can occur near the fuel electrode. These results indeed have been experimentally confirmed. In the work of Laguna-Bercero et al. [11], for example, it was shown that the oxygen concentration in the YSZ electrolyte just under the oxygen electrode was much higher than the starting value (indication of high $\left.\mu_{O_{2}}(x)\right)$ and lower in the electrolyte just under the fuel electrode (indication of low $\mu_{O_{2}}(x)$ ) [11].

Theoretical analysis has demonstrated that the degree to which the oxygen chemical potential inside the electrolyte, $\mu_{O_{2}}(x)$, can go out of bounds depends upon the electronic and the ionic resistances of the electrolyte and the electrolyte/electrode interfaces [1,2]. The analysis shows that the higher the electronic conductivity of the electrolyte, the lower is variation of $\mu_{O_{2}}(x)$ across the electrolyte for a given set of operating conditions. Since $8 \mathrm{YSZ}$ is a purely oxygen ion conductor with very low electronic conductivity, it has a high propensity for developing a large variation (gradient) in $\mu_{O_{2}}(x)$, thus leading to oxygen electrode delamination, internal cracking as well as internal reduction, as observed [11]. If however the electronic conductivity can be increased by doping (while still retaining ionic conductivity as the dominant conductivity) the propensity to degradation may be drastically lowered. The principal objective of the experimental part of the present work, in which stacks made of 8YSZ and 8CYSZ cells were 
tested in both SOFC and SOEC modes, was to verify these predictions. In what follows, the results of long term testing of 8YSZ and 8CYSZ stacks in the SOEC mode are presented.

Figure 7 shows the results of a 5 cell 8 YSZ stack tested in SOEC mode at $800^{\circ} \mathrm{C}$. The fuel electrode gas contained a mixture of $70 \% \mathrm{H}_{2} \mathrm{O}$ and $30 \% \mathrm{H}_{2}$. The average cell voltage was $1.25 \mathrm{~V}$ and the net current was maintained at -14.3 A. Thus, the net electrical power per cell was -14.3 $\mathrm{x} 1.25=-17.88 \mathrm{~W}$. That is, the electrical power supplied to the stack was $17.88 \times 5=89.4 \mathrm{~W}$. The figure shows that the cell voltages were different for different cells, and stable behavior was not observed. All cell voltages increased over time which is consistent with degradation involving internal cracking and/or electrode delamination (both of these processes are expected to occur slowly with time, with the rate of degradation dependent upon the operating conditions). After about 140 h, large changes in cell voltages occurred. After 160 h, the stack failed. After cooling down, it was observed that considerable damage had occurred to the cells including electrode delamination.

Figure 8 shows the results of a 5 cell $8 \mathrm{CYSZ}$ stack tested in SOEC mode at $800^{\circ} \mathrm{C}$. The fuel electrode gas contained a mixture of $70 \% \mathrm{H}_{2} \mathrm{O}$ and $30 \% \mathrm{H}_{2}$. The average cell voltage was $0.92 \mathrm{~V}$ and the net current was maintained at -20.3 A. Thus, the net electrical power per cell was -20.3 x $0.9=-18.27 \mathrm{~W}$. That is, the electrical power supplied to the stack was $18.27 \times 5=91.35 \mathrm{~W}$. This means, the two stacks were operated under approximately the same conditions, but only insofar as the input power was concerned. As stated earlier, in order to realize the same performance characteristics as the 8 YSZ stack, the $8 \mathrm{CYSZ}$ stack would need to be operated at a current of $-46 \mathrm{~A}$, but was operated only at $-20.3 \mathrm{~A}$. 
The 8CYSZ stack, however, was far more stable, while the 8YSZ stack degraded and eventually failed under the operating conditions. The experimental results demonstrated that MIEC cells exhibit stable performance. Further optimization of electrolyte composition is needed to maximize the performance while maintaining stability.

Thermodynamic Basis for the Stability of Cells: In the SOFC mode (single cell only), the ionic and the electronic currents through the cell are anti-parallel. In such a case, the oxygen chemical potential inside the electrolyte, $\mu_{O_{2}}(x)$, is always bounded by values at the electrodes [1,2]. Thus if the electrolyte is stable over the imposed $\Delta \mu_{O_{2}}=\mu_{O_{2}}^{I}-\mu_{O_{2}}^{I I}$, it continues to be stable over the range of operating conditions. In the SOEC mode, however, the ionic and the electronic currents through the electrolyte are parallel. Under such conditions, the $\mu_{O_{2}}(x)$ in the electrolyte can exceed electrode bounds [1,2]. Depending upon the transport properties and the operating conditions, the $\mu_{O_{2}}(x)$ can become very high and/or very low thus increasing the propensity to degradation $[1,2]$. This is consistent with the observations that in general greater degradation is observed in the SOEC mode. Prior work has also shown that the electronic conductivity of the electrolyte plays a major role in dictating the spatial variation of the oxygen chemical potential, $\mu_{O_{2}}(x)$, inside the electrolyte [1,2]. If the $\mu_{O_{2}}(x)$ is too high, cracking and/or oxygen electrode delamination can occur. If the $\mu_{O_{2}}(x)$ is too low local reduction can occur. Both of these situations can occur inside the electrolyte of an operating cell in the SOEC mode. Indeed, results of Laguna-Bercero et al. have shown experimental evidence of this in SOEC made with 8YSZ as the electrolyte [11]. 
In what follows, we examine the theoretical basis founded on linear non-equilibrium thermodynamics and transport to determine under what operating conditions and for what transport properties cells may exhibit better durability. The chemical potential of oxygen inside the electrolyte close to the oxygen electrode is given by [2] (different notations are used in this manuscript compared to that in [2])

$$
\mu_{O_{2}}^{c}=\mu_{O_{2}}^{I}-4 F\left\{\frac{E_{A} R_{e(c)}}{R_{e}}-\frac{\left(E_{A}-E_{N}\right) R_{i(c)}}{R_{i}}\right\}
$$

and the oxygen chemical potential inside the electrolyte close to the fuel electrode is given by

$$
\mu_{O_{2}}^{a}=\mu_{O_{2}}^{I I}+4 F\left\{\frac{E_{A} R_{e(a)}}{R_{e}}-\frac{\left(E_{A}-E_{N}\right) R_{i(a)}}{R_{i}}\right\}
$$

Thus, the difference in the two chemical potentials, which gives the net oxygen chemical potential difference, $\Delta \mu_{\mathrm{O}_{2}}$, in the electrolyte just inside the electrode/electrolyte interfaces, is given by

$$
\mu_{O_{2}}^{c}-\mu_{O_{2}}^{a}=\mu_{O_{2}}^{I}-\mu_{O_{2}}^{I I}-4 F E_{A}\left(\frac{R_{e(c)}+R_{e(a)}}{R_{e}}\right)+4 F\left(E_{A}-E_{N}\right)\left(\frac{R_{i(c)}+R_{i(a)}}{R_{i}}\right)
$$

Note that 


$$
\mu_{O_{2}}^{I}-\mu_{O_{2}}^{I I}=4 F E_{N}
$$

Using equations (1) and (2), note that

$$
\mu_{O_{2}}^{c}-\mu_{O_{2}}^{a}=4 F E_{N}\left(\frac{R_{i(e l)}}{R_{i}}\right)+4 F E_{A}\left(\frac{R_{i(c)}+R_{i(a)}}{R_{i}}\right)-4 F E_{A}\left(\frac{R_{e(c)}+R_{e(a)}}{R_{e}}\right)
$$

Now note that

$$
\begin{aligned}
& \left(\frac{R_{i(e l)}}{R_{i}}\right)<1 \\
& \left(\frac{R_{i(c)}+R_{i(a)}}{R_{i}}\right)<1
\end{aligned}
$$

and

$$
\left(\frac{R_{e(c)}+R_{e(a)}}{R_{e}}\right)<1
$$

Various possibilities exist depending upon the relative values of the ionic and the electronic resistances as well as the applied voltage, $E_{A}$. We will consider a few illustrative cases.

Case I: The electrolyte is a purely ionic conductor such that 


$$
R_{e}>>R_{i}
$$

and we also assume

$$
R_{e(e l)}>R_{e(c)}, R_{e(a)}
$$

Equation (116) means that on a relative basis we are assuming the resistances to the transport of electrons just across electrode/electrolyte interfaces are much lower than the electronic resistance of the electrolyte. In such a case,

$$
\left(\frac{R_{e(c)}+R_{e(a)}}{R_{e}}\right)<<1
$$

and the chemical potential difference across the electrolyte just inside the electrode/electrolyte interfaces, $\Delta \mu_{O_{2}}$, is given by

$$
\mu_{O_{2}}^{c}-\mu_{O_{2}}^{a} \approx 4 F E_{N}\left(\frac{R_{i(e l)}}{R_{i}}\right)+4 F E_{A}\left(\frac{R_{i(c)}+R_{i(a)}}{R_{i}}\right)
$$

or

$$
\mu_{O_{2}}^{c}-\mu_{O_{2}}^{a} \approx 4 F E_{N}+4 F\left(E_{A}-E_{N}\right)\left(\frac{R_{i(c)}+R_{i(a)}}{R_{i}}\right)
$$


Thus, depending upon the magnitude of the applied voltage, the ionic resistance of the electrolyte and the polarization resistances, for $E_{A}>E_{N}$ (SOEC)

$$
\mu_{O_{2}}^{c}-\mu_{O_{2}}^{a}>4 F E_{N}
$$

or

$$
\mu_{O_{2}}^{c}-\mu_{O_{2}}^{a}>\mu_{O_{2}}^{I}-\mu_{O_{2}}^{I I}
$$

This is especially the case if the polarization resistances are high (poor quality electrodes). Thus, under some conditions, the chemical potential variation in the electrolyte will be larger than that imposed across the two electrodes, the latter being $\mu_{O_{2}}^{I}-\mu_{O_{2}}^{I I}$. Figure 9(a) shows a schematic.

High oxygen pressures may develop just under the oxygen electrode to cause delamination and low oxygen partial pressures may develop just under the fuel electrode to cause local reduction. This is the likely scenario with YSZ as the electrolyte because of its very low electronic conductivity [11].

Note that if $R_{i(e l)}<<R_{i(a)}, R_{i(c)}$, which means high electrode polarization resistances relative to the electrolyte ionic resistance, then equation (119) reduces to

$$
\mu_{O_{2}}^{c}-\mu_{O_{2}}^{a} \approx 4 F E_{A}
$$

Thus, when the applied voltage exceeds the Nernst voltage, that is when $E_{A}>E_{N}$, which is the case in the SOEC mode, large variation in oxygen chemical potential inside the electrolyte occurs. Thus high polarization resistances are detrimental. This is a severe condition and cell 
degradation is expected. Also note that the higher the applied voltage, the greater the $\mu_{O_{2}}^{c}-\mu_{O_{2}}^{a}$, and thus the greater is the tendency for degradation as is well known.

The results on long term test on 8 YSZ stack given in Figure 7 can now be examined in light of equation (122). As stated earlier, this stack had a very high overall resistance in the SOEC mode. Thus, we expect $R_{i(c)}+R_{i(a)} \gg R_{i(e l)}$. Thus the chemical potential difference within the 8YSZ cells is expected to be given by equation (122), that is governed by the applied voltage, $E_{A}$. The applied voltage per cell was $E_{A}=1.25 \mathrm{~V}$. The oxygen partial pressure in the fuel gas, $p_{\mathrm{O}_{2}}^{I I}$, was $\sim 1.5 \times 10^{-17}$ atm. If we assume the $p_{\mathrm{O}_{2}}^{a}$ to be the same, then the corresponding $p_{\mathrm{O}_{2}}^{c}$ is estimated to be over $4 \times 10^{6}$ atm. However, the actual $p_{\mathrm{O}_{2}}^{a}$ will likely be much lower than $p_{\mathrm{O}_{2}}^{I I}$. Thus, we expect the $p_{\mathrm{O}_{2}}^{c}$ proportionately lower, yet may be several hundred atmospheres or even several thousand atmospheres. Thus, oxygen electrode delamination in $8 Y S Z$ stacks is deemed inevitable. The observed degradation and eventual failure of the $8 \mathrm{YSZ}$ stacks tested in the present study (Figure 7) is in accord with this expectation.

On the other hand, if the polarization resistances are negligible (highly active electrodes) such that $R_{i(e l)}>>R_{i(a)}, R_{i(c)}$, then from equation (119) note that

$$
\mu_{O_{2}}^{c}-\mu_{O_{2}}^{a} \approx 4 F E_{N}
$$


That is, the difference in oxygen chemical potential within the electrolyte will be the same as the applied chemical potential difference, $\mu_{O_{2}}^{I}-\mu_{O_{2}}^{I I}$, and essentially independent of the applied voltage, $E_{A}$. This means in principle it may be possible to develop SOEC capable of operating at high voltages even with a purely ion conducting electrolyte such as YSZ, provided the electrodes are highly active. This, however, must be achieved by lowering the polarization resistance while maintaining low enough overall ionic resistance of the cell, $R_{i}$, to ensure good performance.

Case II: The electrolyte is an MIEC such that

$$
R_{e}=R_{e(c)}+R_{e(a)}+R_{e(e l)} \approx R_{e(c)}+R_{e(a)}
$$

which means

$$
R_{e(c)}+R_{e(a)}>R_{e(e l)}
$$

This could be the case wherein almost all of the electronic resistance is associated with electrolyte/electrode interfaces. Such would be the case with an MIEC electrolyte. The difference in oxygen chemical potentials is now given by

$$
\mu_{O_{2}}^{c}-\mu_{O_{2}}^{a} \approx 4 F E_{N}\left(\frac{R_{i(e l)}}{R_{i}}\right)+4 F E_{A}\left(\frac{R_{i(c)}+R_{i(a)}}{R_{i}}\right)-4 F E_{A}
$$

or 


$$
\mu_{O_{2}}^{c}-\mu_{O_{2}}^{a} \approx 4 F\left(E_{N}-E_{A}\right)\left(\frac{R_{i(e l)}}{R_{i}}\right)
$$

Thus, in such a case,

$$
\mu_{O_{2}}^{c}<\mu_{O_{2}}^{a}
$$

in the SOEC mode $\left(E_{A}>E_{N}\right)$. Returning to equation (109), we note the following situation when the electrodes are highly active and the electrolyte is an MIEC. Specifically, let us consider the case when $R_{i(e l)}>R_{i(a)}, R_{i(c)}$ (electrode polarization resistances are very small - highly active electrodes) and when $R_{e(c)}+R_{e(a)} \gg R_{e(e l)}$ (electrolyte is an MIEC and much of the cell electronic resistance is associated with electrode/electrolyte interfaces), then equation (109) reduces to

$$
\mu_{O_{2}}^{c}-\mu_{O_{2}}^{a} \approx \mu_{O_{2}}^{I}-\mu_{O_{2}}^{I I}-4 F E_{A}=4 F\left(E_{N}-E_{A}\right)
$$

The chemical potential within the electrolyte does not exceed bounds at small applied voltages. Figure 9(b) shows a schematic. Note that in this case the $\mu_{O_{2}}(x)$ increases along $x$ (from oxygen electrode to fuel electrolyte). The cell behaves in a stable manner at small applied voltages. This is the case with $8 \mathrm{CYSZ}$ cells in the present work. The preceding shows that relative magnitudes of electronic and ionic resistances determine the variation of $\mu_{O_{2}}(x)$ within the electrolyte. By appropriate design of an MIEC electrolyte, it should be possible to minimize the variation of 
$\mu_{O_{2}}(x)$ in the electrolyte leading to more durable cells. At the same time, as the ionic and electronic currents are parallel in the SOEC mode, there can be conditions under which very high and/or very low oxygen chemical potential occur within the cell. Thus, degradation of SOEC is more likely than of SOFC. However, by proper design of cell transport properties (electrolyte and electrodes), the incidence of cell degradation can in principle be minimized.

\section{Summary}

In this manuscript, theoretical analysis based on simple equivalent circuits for SOEC and SOFC operating in steady state was presented for purely ionic conducting electrolyte cells and MIEC electrolyte cells. The selected electronic conductivity was substantial (corresponding to an ionic transference number of $\sim 0.9$ ). Thus, during both modes of operation, the electronic current flowing through the cell was significant. It was shown that by a suitable choice of cell parameters (ionic and electronic resistances) it is possible to design MIEC cells capable of exhibiting the same performance characteristics as cells made with purely ionic conducting electrolyte cells. The results show that it is not necessary that the electrolyte be a purely ionic conductor (transference number close to 1) to realize efficient operation in both the SOFC and the SOEC modes. The MIEC cells (SOEC mode) exhibit more stable operation than cells made with purely ionic conducting electrolyte cells. Specifically, the incidence of degradation of cells by oxygen electrode delamination is substantially reduced when using MIEC electrolyte cells. Experimental results are presented on 5 cell stacks made of $8 \mathrm{YSZ}$ electrolyte cells and cells 
made with $8 \mathrm{YSZ}$ cells doped with $8 \% \mathrm{CeO}_{2}$ (8CYSZ), which was added to increase the electronic conductivity of the electrolyte. The 8YSZ stacks in the SOEC mode exhibited unstable operation and considerable degradation over the duration of the test $(140 \mathrm{~h})$ and the stack failed after $\sim 160$ h. A sharp drop in voltage occurred at the end of the test. By contrast, the 8CYSZ stack exhibited very stable operation over the duration of the test $(140 \mathrm{~h})$ and there was no evidence of stack degradation.

The observed stable operation of MIEC cells is consistent with our prior theoretical and experimental studies which showed that the stability of the electrolyte (and thus of the cells) depends upon some fundamental considerations of non-equilibrium thermodynamics and transport $[1,2]$. Specifically, it was shown that the electronic conductivity in predominantly ionic conductors (e.g. YSZ) is important concerning the thermodynamics of the electrolyte under transport, mainly the spatial distribution of oxygen chemical potential, $\mu_{O_{2}}(x)$, inside the electrolyte. In addition, electrode polarizations play an important role in governing the spatial variation of $\mu_{O_{2}}(x)$ through the electrolyte. If the electronic conductivity is very low such as in YSZ and electrode polarizations are large, large variations in $\mu_{O_{2}}(x)$ can occur and the $\mu_{O_{2}}(x)$ inside the electrolyte may exceed electrode bounds. The resulting very high or very low values of $\mu_{O_{2}}(x)$ can lead to electrode delamination, local electrolyte cracking and local electrolyte decomposition. However, the $\mu_{O_{2}}(x)$ exhibits lower spatial variation if the electronic conductivity is relatively high (with the ionic conductivity still being the dominant one). Under suitable conditions, the $\mu_{O_{2}}(x)$ in the electrolyte can be maintained within the electrode bounds, thus preventing/suppressing electrolyte and thus cell degradation. Cells made with 8CYSZ electrolyte had an ionic transference number of $\sim 0.9$ and could be operated stably. Many other 
electrolytes and dopants are possible for the development of stable cells operating in both the SOFC and the SOEC modes. The development of new electrolyte compositions with optimized levels of electronic conductivity and the development of highly active electrodes could be very beneficial from the standpoint of SOFC and SOEC long term durability without compromising performance characteristics.

Acknowledgement: The modeling part of this work (A. V. Virkar) was supported at the University of Utah by the U. S. Department of Energy under Grant Number DE-FG0206ER46086. The experimental part of this work (stack tests, G. Tao) was supported at Materials and Systems Research, Inc. by Idaho National Laboratory. 


\section{References}

1) Virkar AV. Theoretical analysis of the role of interfaces in transport through oxygen ion and electron conducting membranes. J Power Sources 2005; 147 (1-2):8-31.

2) Virkar AV. Mechanism of oxygen electrode delamination in solid oxide electrolyzer cells. Int J Hydrogen Energy 2010; 35(18):9527-43.

3) Lim HT, Virkar AV, Electrochemical degradation of fuel cells: effect of electrolyte composition. SOFC IX, ECS Trans 2009; 25(2):447-56.

4) Virkar AV. Theoretical analysis of solid oxide fuel cells with two-layer, composite electrolyte: electrolyte stability. J Electrochem Soc 1991; 138(5): 1481-87.

5) Lim HT, Virkar AV. Measurement of oxygen chemical potential in $\mathrm{Gd}_{2} \mathrm{O}_{3}$-doped ceria$\mathrm{Y}_{2} \mathrm{O}_{3}$-stabilized zirconia bi-layer electrolyte, anode-supported solid oxide fuel cells. $\mathrm{J}$ Power Sources 2009; 192:267-78.

6) Heyne L. Mass transport in oxides. National Bureau of Standards Special Publication 296, Edited by Wachtman JB, Franklin AD, 1968; p. 149.

7) Hasse R. Thermodynamics of irreversible processes. New York: Dover Publications; 1969.

8) Kondepudi D, Prigogine I. Modern thermodynamics: From heat engines to dissipative structures. New York.: John Wiley and Sons; 1998.

9) Kjelstrup S, Bedeaux D. Non-Equilibrium thermodynamics of heterogeneous systems. New Jersey: World Scientific; 2008.

10) O'Brien JE, Stoots CM, Herring JS, Condie KG, Housley GK. The high temperature electrolysis program at the Idaho National Laboratory: observations on performance degradation. OSTI ID: 96915, DE-AC07-99ID-13727. Report no. INL/CON-09-15564.

11) Laguna-Bercero MA, Campana R, Larrea A, Kilner JA, Orera VM. Electrolyte degradation in anode supported microtubular yttria-stabilized zirconia-based solid oxide steam electrolysis cells at high voltage of operation. J. Power Sources 2011; 196(21):8942-7. 


\section{Figure Captions:}

Figure 1: An equivalent circuit for a cell operating in steady state. The load is described by an external voltage source (battery). Concentration polarization is assumed to be small.

Figure 2: An equivalent circuit in steady state. All ionic resistances of the cell are lumped into $R_{i}$ and all electronic resistances into $R_{e}$. If $E_{A}<E_{N}$, the cell is an SOFC; if $E_{A}>E_{N}$, the cell is an SOEC.

Figure 3(a): An equivalent circuit in steady state for a cell made of a purely ionic conducting electrolyte; $R_{e(1)} \gg R_{i(1)}$. The electronic resistance can be ignored in the calculations of performance characteristics (but must be taken into account to determine the position dependence of the oxygen chemical potential, $\left.\mu_{O_{2}}(x)\right)$.

Figure 3(b): An equivalent circuit in steady state for a cell made with an MIEC electrolyte. The electronic resistance cannot be ignored for either the calculations of performance characteristics or the chemical potential of oxygen, $\mu_{O_{2}}(x)$.

Figure 4: Plots of the voltage vs. current for the two cells. The crossover point corresponds to $E_{A}=0.7364 \mathrm{~V}$ and $I_{L}=0.3273 \mathrm{Acm}^{-2}$. Also shown in the figure are points (green dots) corresponding to identical performance characteristics in the SOEC mode and the SOFC mode. 
Figure 5: Performance curves for a 5 cell stack made of 8YSZ electrolyte tested in SOEC and SOFC modes. For SOEC tests, the compositions of the fuel gas mixtures were as follows: $90 \%$ $\mathrm{H}_{2} \mathrm{O}+10 \% \mathrm{H}_{2}, 80 \% \mathrm{H}_{2} \mathrm{O}+20 \% \mathrm{H}_{2}, 70 \% \mathrm{H}_{2} \mathrm{O}+30 \% \mathrm{H}_{2}$, and $50 \% \mathrm{H}_{2} \mathrm{O}+50 \% \mathrm{H}_{2}$. For SOFC tests, the compositions of the gaseous mixtures were as follows: $\sim 100 \% \mathrm{H}_{2}, 90 \% \mathrm{H}_{2}+10 \% \mathrm{H}_{2} \mathrm{O}$, $70 \% \mathrm{H}_{2}+30 \% \mathrm{H}_{2} \mathrm{O}$, and $50 \% \mathrm{H}_{2}+50 \% \mathrm{H}_{2} \mathrm{O}$. The green dots on the extrapolated lines correspond to conditions under which the 8YSZ and 8CYSZ stacks exhibit identical performance characteristics.

Figure 6: Performance curves for a 5 cell stack made of 8 YSZ electrolyte doped with 8 mol.\% $\mathrm{CeO}_{2}$ electrolyte (8CYSZ) tested in SOEC and SOFC modes. For SOEC tests, the compositions of the fuel gas mixtures were as follows: $80 \% \mathrm{H}_{2} \mathrm{O}+20 \% \mathrm{H}_{2}, 70 \% \mathrm{H}_{2} \mathrm{O}+30 \% \mathrm{H}_{2}$, and $50 \%$ $\mathrm{H}_{2} \mathrm{O}+50 \% \mathrm{H}_{2}$. For SOFC tests, the compositions of the gaseous mixtures were as follows: $\sim 100 \% \mathrm{H}_{2}, 70 \% \mathrm{H}_{2}+30 \% \mathrm{H}_{2} \mathrm{O}$, and $50 \% \mathrm{H}_{2}+50 \% \mathrm{H}_{2} \mathrm{O}$. The green dots on the extrapolated lines correspond to conditions under which the 8YSZ and 8CYSZ stacks exhibit identical performance characteristics.

Figure 7: Long term $(160 \mathrm{~h})$ test at $800^{\circ} \mathrm{C}$ of a 5 cell stack made of 8 YSZ electrolyte in SOEC mode. The stack current was maintained at -14.3 A. The average voltage per cell (repeat unit) was $1.25 \mathrm{~V}$. The stack did not exhibit stable behavior. After $140 \mathrm{~h}$, large changes in cell voltages occurred. The stack failed after $160 \mathrm{~h}$. 
Figure 8: Long term $(135 \mathrm{~h})$ test at $800^{\circ} \mathrm{C}$ of a 5 cell stack made of $8 \mathrm{CYSZ}$ electrolyte in SOEC mode at $800^{\circ} \mathrm{C}$. The stack current was maintained at $-20.3 \mathrm{~A}$. The average cell voltage was $0.9 \mathrm{~V}$. All cell voltages were nearly the same and remained stable throughout the test.

Figure 9: Schematics of the two cases. (a) Case I: The electronic conductivity of the electrolyte (e.g. YSZ) is very low. The $\Delta \mu_{O_{2}}$ is out of bounds, and cell degradation may occur. (b) Case II: The electrolyte is an MIEC (e.g. 8CYSZ). The $\Delta \mu_{O_{2}}$ is bounded for the case shown. The cell is stable and resistant to degradation. 


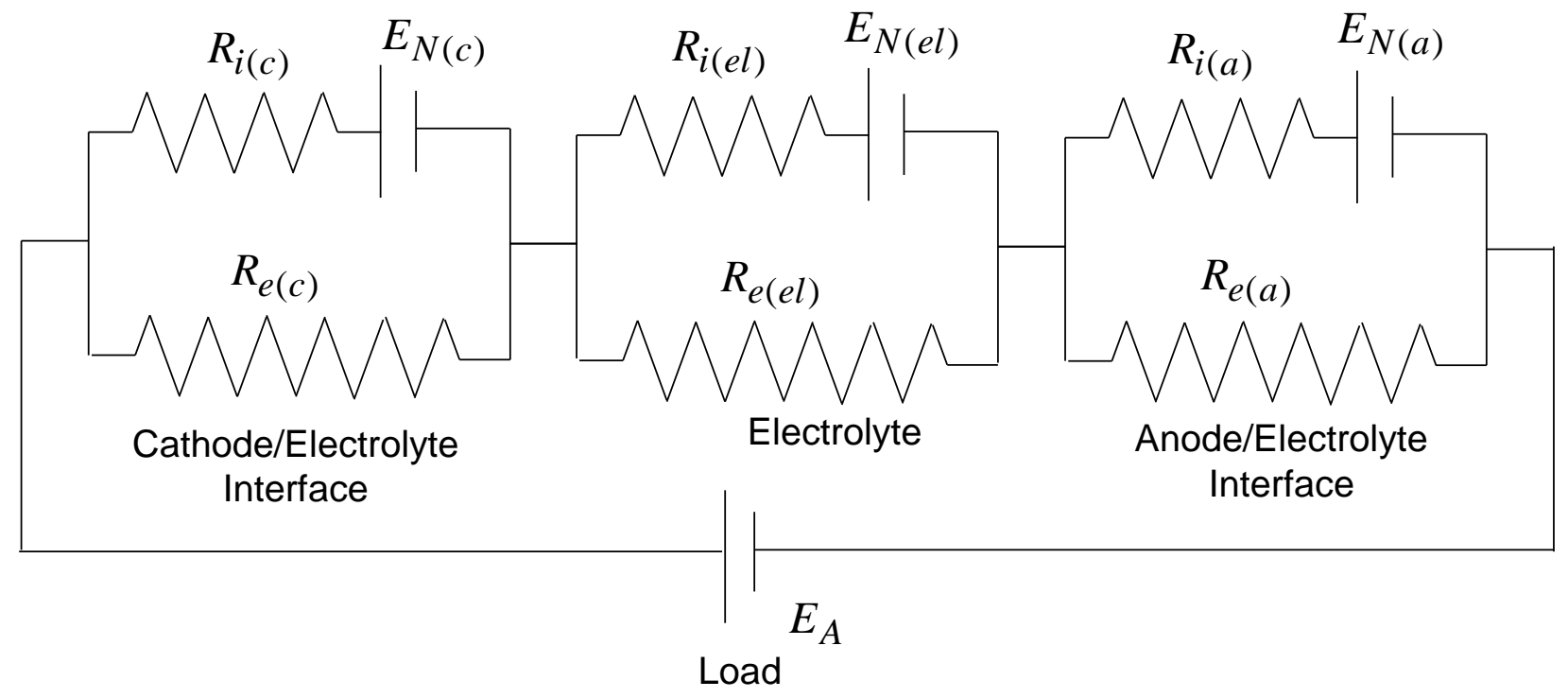

Figure 1: An equivalent circuit for a cell operating in steady state. The load is described by an external voltage source (battery). Concentration polarization is assumed to be small. 


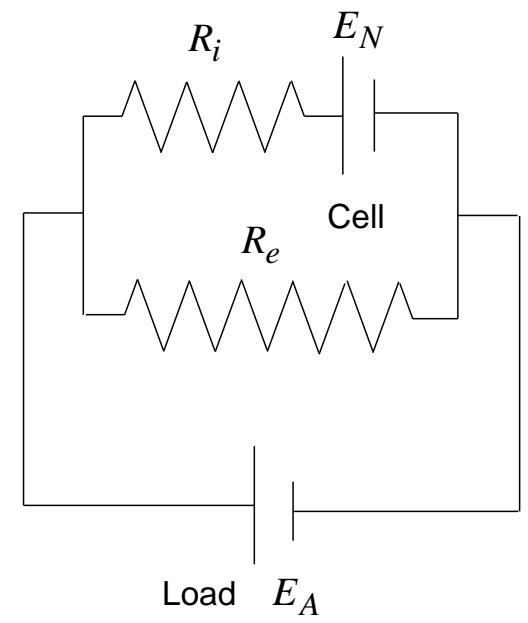

Figure 2: An equivalent circuit in steady state. All ionic resistances of the cell are lumped into $R_{i}$ and all electronic resistances into $R_{e}$. If $E_{A}<E_{N}$, the cell is an SOFC; if $E_{A}>E_{N}$, the cell is an SOEC. 


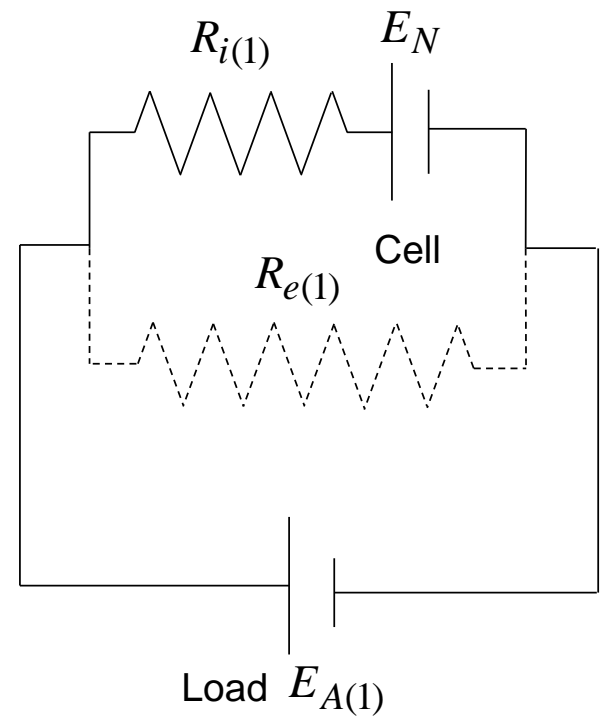

Figure 3(a): An equivalent circuit in steady state for a cell made of a purely ionic conducting electrolyte; $R_{e(1)} \gg R_{i(1)}$. The electronic resistance can be ignored in the calculations of performance characteristics (but must be taken into account to determine the position dependence of the oxygen chemical potential, $\mu_{O_{2}}(x)$ ). 


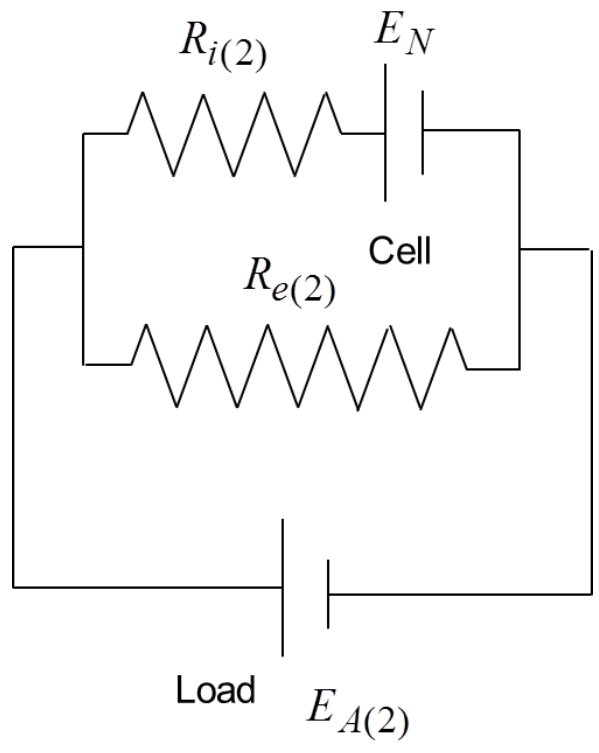

Figure 3(b): An equivalent circuit in steady state for a cell made with an MIEC electrolyte. The electronic resistance cannot be ignored for either the calculations of performance characteristics or the chemical potential of oxygen, $\mu_{O_{2}}(x)$. 


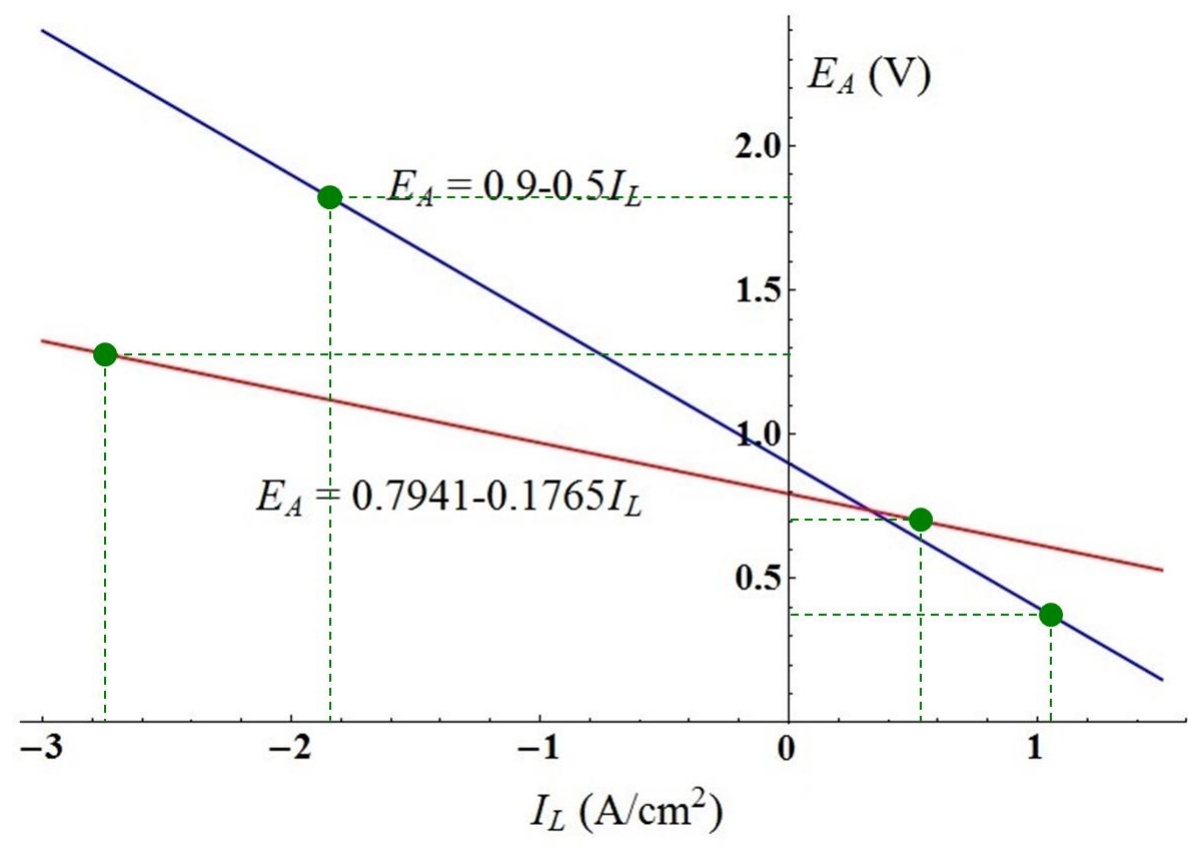

Figure 4: Plots of the voltage vs. current for the two cells. The crossover point corresponds to $E_{A}=0.7364 \mathrm{~V}$ and $I_{L}=0.3273 \mathrm{Acm}^{-2}$. Also shown in the figure are points (green dots) corresponding to identical performance characteristics in the SOEC mode and the SOFC mode. 


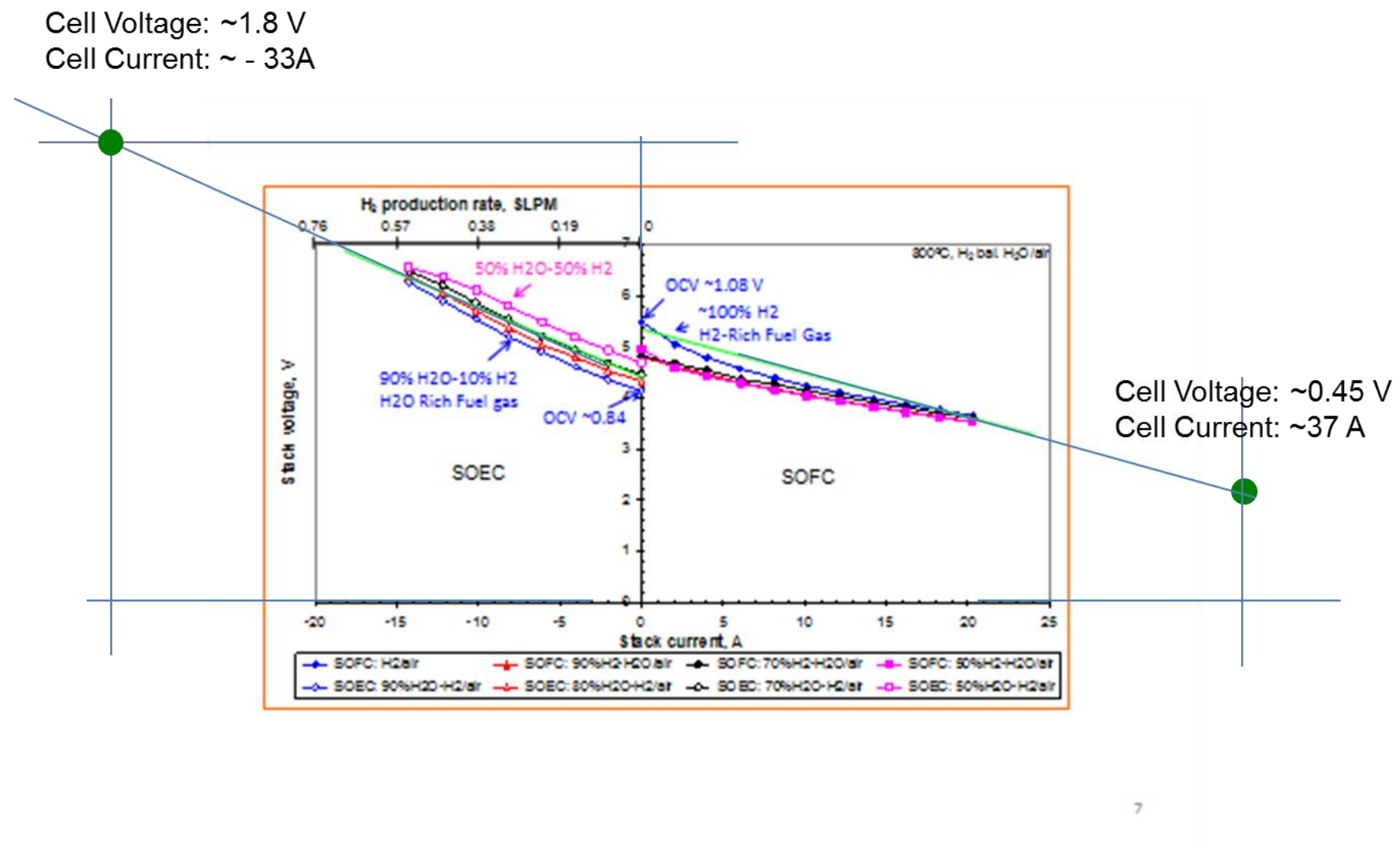

Figure 5: Performance curves for a 5 cell stack made of 8YSZ electrolyte tested in SOEC and SOFC modes. For SOEC tests, the compositions of the fuel gas mixtures were as follows: $90 \%$ $\mathrm{H}_{2} \mathrm{O}+10 \% \mathrm{H}_{2}, 80 \% \mathrm{H}_{2} \mathrm{O}+20 \% \mathrm{H}_{2}, 70 \% \mathrm{H}_{2} \mathrm{O}+30 \% \mathrm{H}_{2}$, and $50 \% \mathrm{H}_{2} \mathrm{O}+50 \% \mathrm{H}_{2}$. For SOFC tests, the compositions of the gaseous mixtures were as follows: $\sim 100 \% \mathrm{H}_{2}, 90 \% \mathrm{H}_{2}+10 \% \mathrm{H}_{2} \mathrm{O}$, $70 \% \mathrm{H}_{2}+30 \% \mathrm{H}_{2} \mathrm{O}$, and $50 \% \mathrm{H}_{2}+50 \% \mathrm{H}_{2} \mathrm{O}$. The green dots on the extrapolated lines correspond to conditions under which the $8 \mathrm{YSZ}$ and $8 \mathrm{CYSZ}$ stacks exhibit identical performance characteristics. 


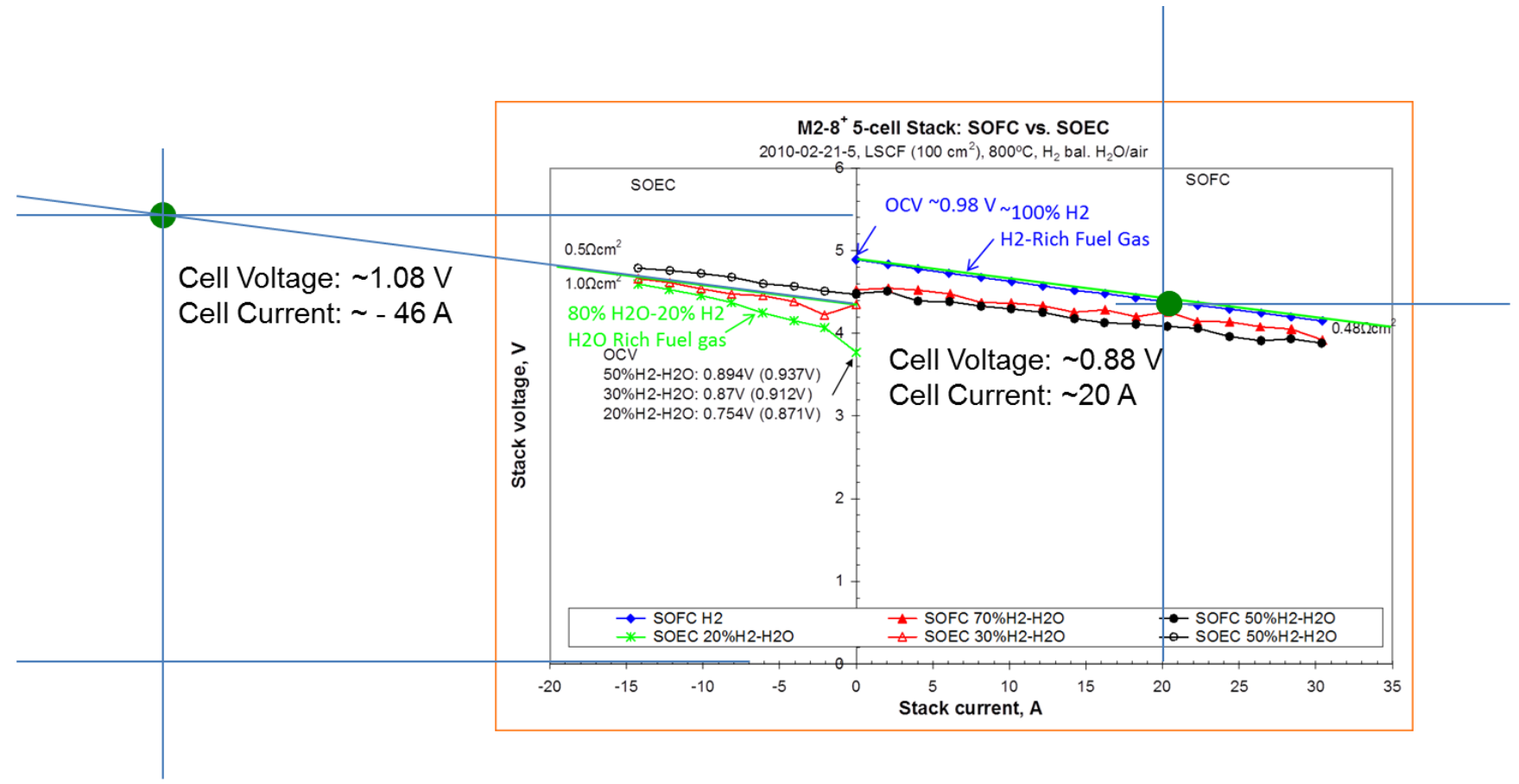

Figure 6: Performance curves for a 5 cell stack made of 8YSZ electrolyte doped with 8 mol.\% $\mathrm{CeO}_{2}$ electrolyte (8CYSZ) tested in SOEC and SOFC modes. For SOEC tests, the compositions of the fuel gas mixtures were as follows: $80 \% \mathrm{H}_{2} \mathrm{O}+20 \% \mathrm{H}_{2}, 70 \% \mathrm{H}_{2} \mathrm{O}+30 \% \mathrm{H}_{2}$, and $50 \%$ $\mathrm{H}_{2} \mathrm{O}+50 \% \mathrm{H}_{2}$. For SOFC tests, the compositions of the gaseous mixtures were as follows: $\sim 100 \% \mathrm{H}_{2}, 70 \% \mathrm{H}_{2}+30 \% \mathrm{H}_{2} \mathrm{O}$, and $50 \% \mathrm{H}_{2}+50 \% \mathrm{H}_{2} \mathrm{O}$. The green dots on the extrapolated lines correspond to conditions under which the 8YSZ and 8CYSZ stacks exhibit identical performance characteristics. 


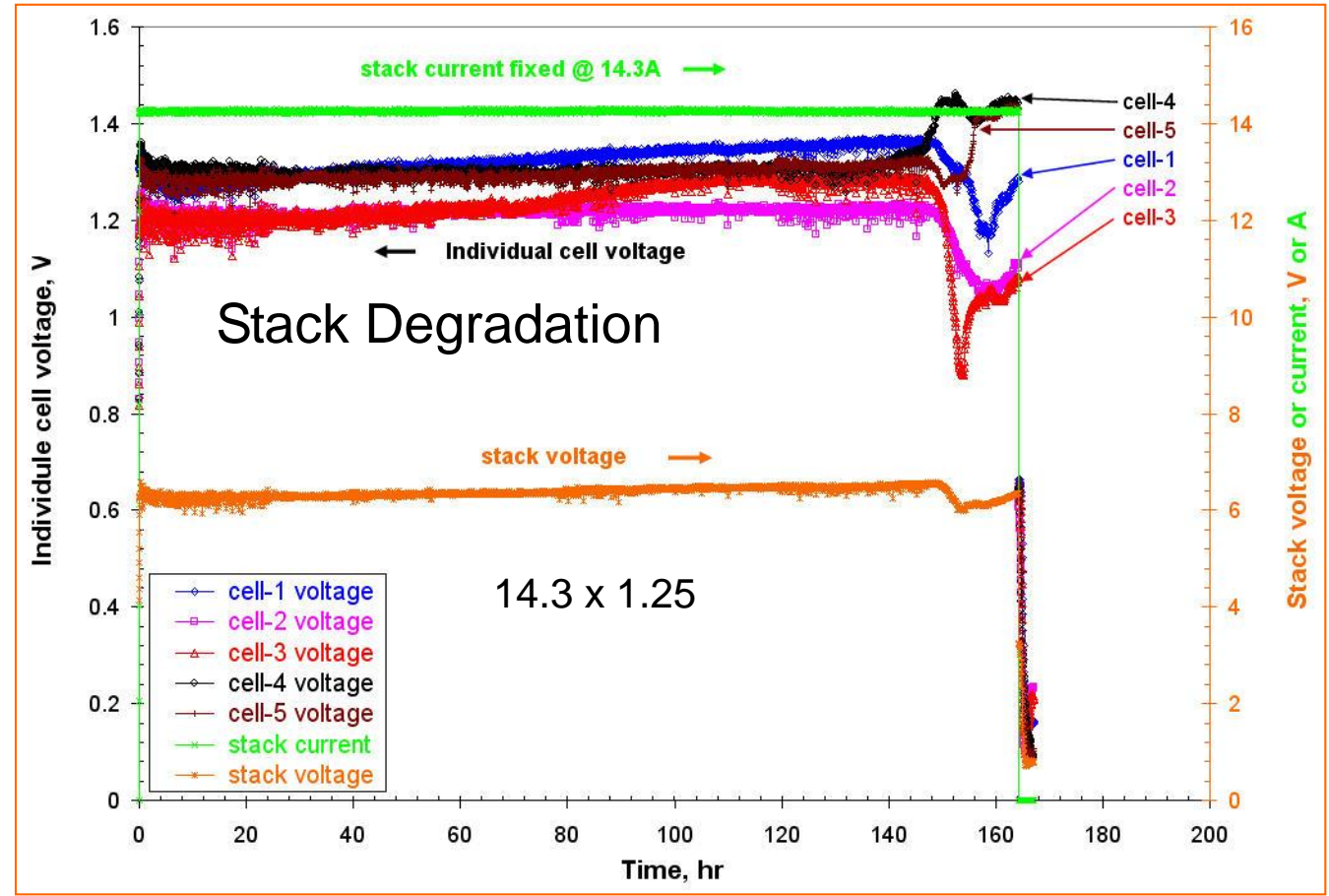

Figure 7: Long term $(160 \mathrm{~h})$ test at $800^{\circ} \mathrm{C}$ of a 5 cell stack made of 8 YSZ electrolyte in SOEC mode. The stack current was maintained at $-14.3 \mathrm{~A}$. The average voltage per cell (repeat unit) was $1.25 \mathrm{~V}$. The stack did not exhibit stable behavior. After $140 \mathrm{~h}$, large changes in cell voltages occurred. The stack failed after $160 \mathrm{~h}$. 


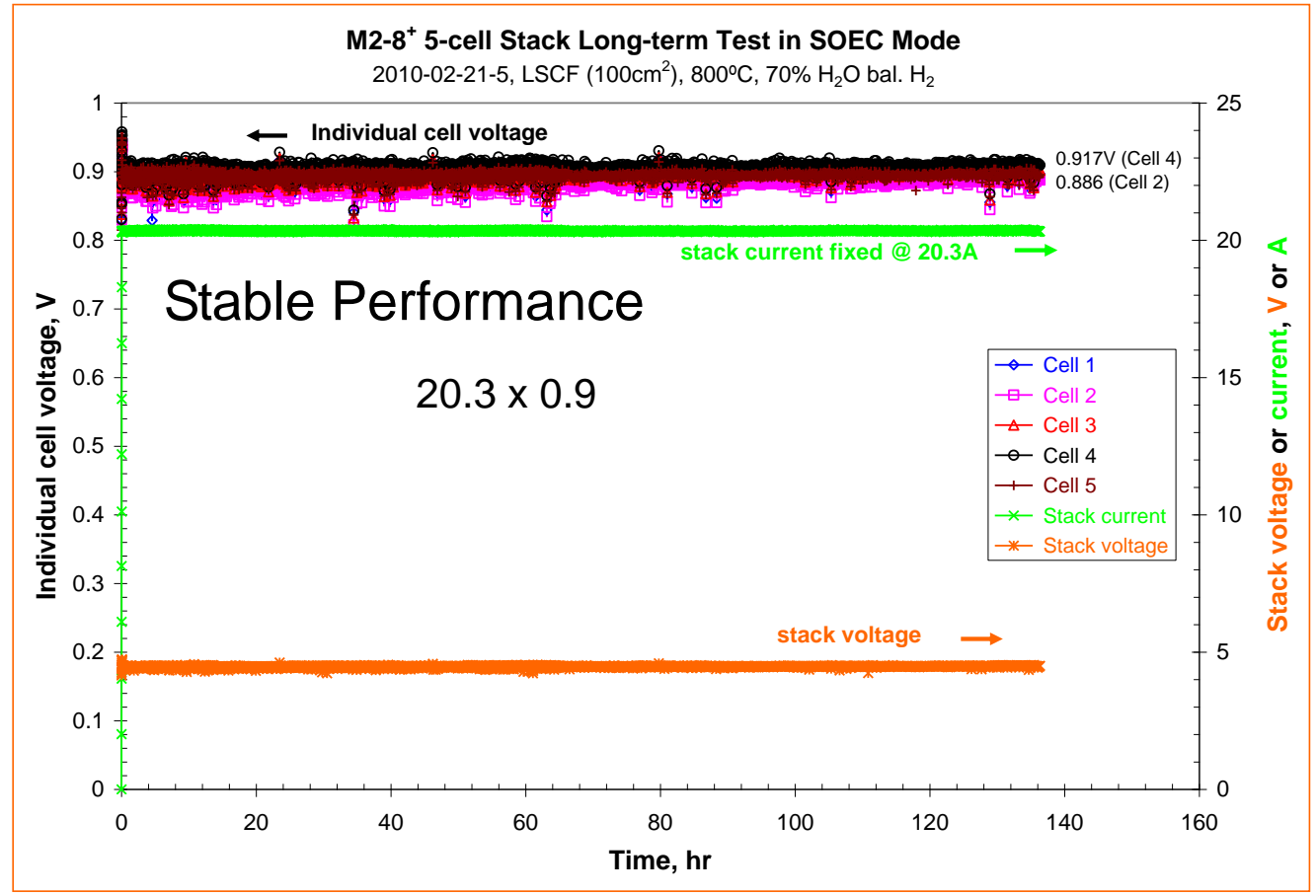

Figure 8: Long term $(135 \mathrm{~h})$ test at $800^{\circ} \mathrm{C}$ of a 5 cell stack made of $8 \mathrm{CYSZ}$ electrolyte in SOEC mode at $800^{\circ} \mathrm{C}$. The stack current was maintained at $-20.3 \mathrm{~A}$. The average cell voltage was $0.9 \mathrm{~V}$. All cell voltages were nearly the same and remained stable throughout the test. 


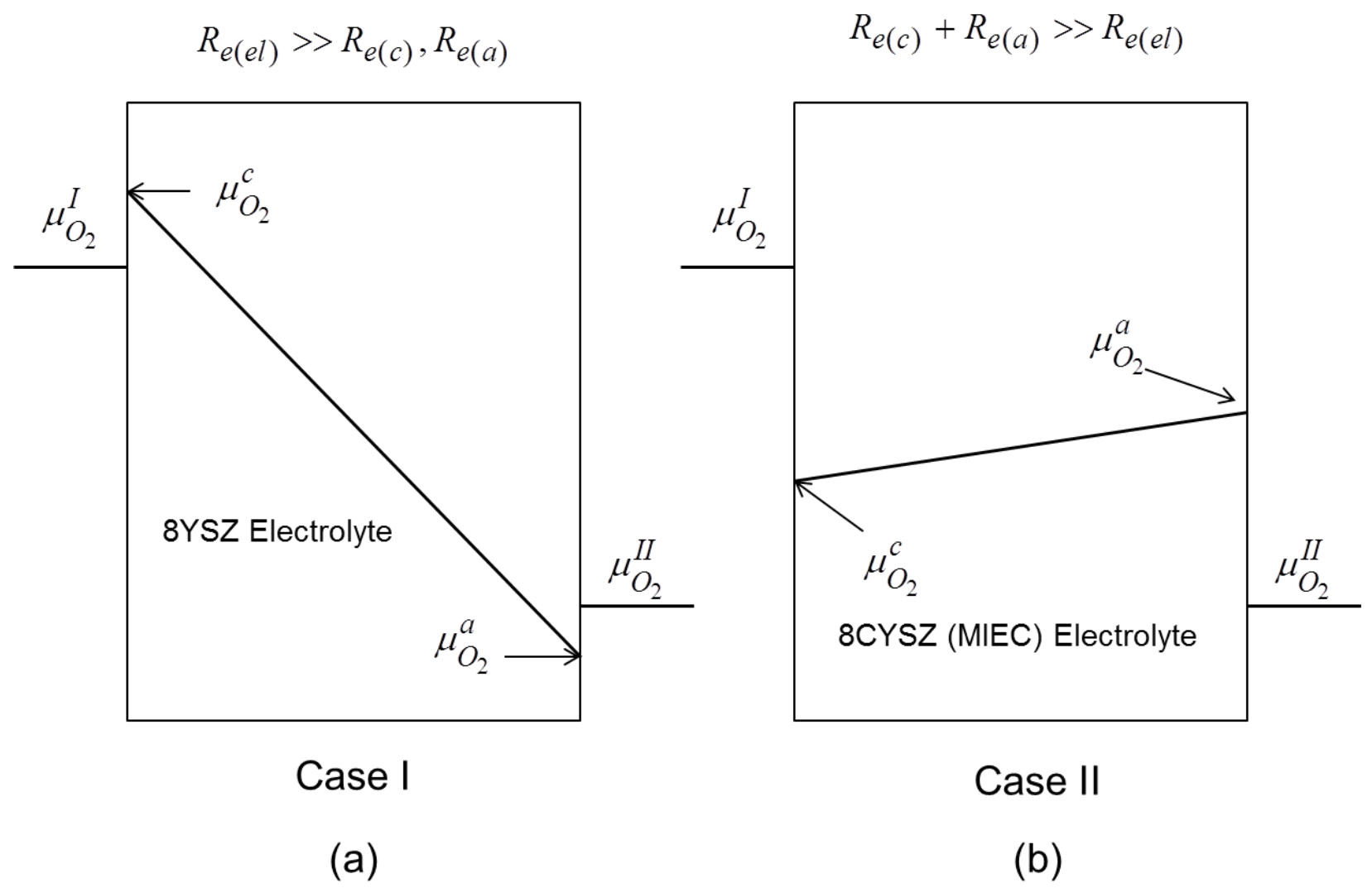

Figure 9: Schematics of the two cases. (a) Case I: The electronic conductivity of the electrolyte (e.g. YSZ) is very low. The $\Delta \mu_{O_{2}}$ is out of bounds, and cell degradation may occur. (b) Case II: The electrolyte is an MIEC (e.g. 8CYSZ). The $\Delta \mu_{O_{2}}$ is bounded for the case shown. The cell is stable and resistant to degradation. 